九州大学学術情報リポジトリ

Kyushu University Institutional Repository

\title{
Numerical study on transient harbor oscillations induced by successive solitary waves
}

Gao, Junliang

School of Naval Architecture and Ocean Engineering, Jiangsu University of Science and Technology

Ji, Chunyan

School of Naval Architecture and Ocean Engineering, Jiangsu University of Science and Technology

Liu, Yingy i

Research Institute for Applied Mechanics, Kyushu University

Ma, Xiaojian

School of Naval Architecture and Ocean Engineering, Jiangsu University of Science and Technology

他

http://hdl. hand le. net/2324/4060501

出版情報：Ocean Dynamics. 68 (2)，pp.193-209，2017-11-27. Springer Nature バージョン：

権利関係 : 
Numerical study on transient harbor oscillations induced by successive solitary waves

$$
\text { Junliang Gao a, b, c, *, Chunyan Ji }{ }^{\text {a }} \text {, Yingyi Liu }{ }^{\mathrm{d}} \text {, Xiaojian Ma }{ }^{\mathrm{a}} \text {, Oleg Gaidai }{ }^{\mathrm{a}}
$$

a School of Naval Architecture and Ocean Engineering, Jiangsu University of Science and Technology, Zhenjiang 212003, China

b Jiangsu Key Laboratory of Coast Ocean Resources Development and Environment Security, Hohai University, Nanjing 210098, China

c State Key Laboratory of Coastal and Offshore Engineering, Dalian University of Technology, Dalian 116024, China

d Research Institute for Applied Mechanics, Kyushu University, Kasuga, Fukuoka 816-8580, Japan

\section{Abstract:}

Tsunamis are traveling waves which are characterized by long wavelengths and large amplitudes close to the shore. Due to the transformation of tsunamis, undular bores have been frequently observed in the coastal zone and can be viewed as a sequence of solitary waves with different wave heights and different separation distances among them.

In this article, transient harbor oscillations induced by incident successive solitary waves are first investigated. The transient oscillations are simulated by a fully nonlinear Boussinesq model, FUNWAVE-TVD. The incident successive solitary waves include double solitary waves and triple solitary waves. This paper mainly focuses on the effects of different waveform parameters of the incident successive solitary waves on the relative wave energy distribution inside the harbor. These wave parameters include the incident wave height, the relative separation distance between adjacent crests and the number of elementary solitary waves in the incident wave train. The relative separation distance between adjacent crests is defined as the ratio of the distance between adjacent crests in the incident wave train to the effective wavelength of the single solitary wave. Maximum oscillations inside the harbor excited by various incident waves are also discussed. For comparison, the transient oscillation excited by the single solitary wave is also considered. The

\footnotetext{
${ }^{*}$ Corresponding author at: School of Naval Architecture and Ocean Engineering, Jiangsu University of Science and Technology, Zhenjiang 212003, China. E-mail address: gaojunliang880917@163.com (J. Gao).
} 
harbor used in this paper is assumed to be long and narrow and has constant depth; the free surface movement inside the harbor is essentially one-dimensional.

This study reveals that, for the given harbor and for the variation ranges of all the waveform parameters of the incident successive solitary waves studied in this paper, the larger incident wave heights and the smaller number of elementary solitary waves in the incident tsunami lead to a more uniform relative wave energy distribution inside the harbor. For the successive solitary waves, the larger relative separation distance between adjacent crests can cause more obvious fluctuations of the relative wave energy distribution over different resonant modes. When the wave height of the elementary solitary wave in the successive solitary waves equals to that of the single solitary wave and the relative separation distance between adjacent crests is equal to or greater than 0.6 , the maximum oscillation inside the harbor induced by the successive solitary waves is almost identical to that excited by the single solitary wave.

\section{Keywords:}

Harbor oscillations; Resonance; Tsunamis; Successive solitary waves; Wave energy distribution; Maximum runup; FUNWAVE-TVD

\section{Introduction}

Harbor oscillations are originated by the trapping and amplification of wave energy at the eigenfrequencies of coastal semi-enclosed water bodies, such as bays and harbors. They may be induced by short wave groups, infragravity waves, tsunamis, wind and pressure fluctuations and shear flow traveling into bays or harbors (Bellotti and Franco, 2011; Bowers, 1977; Chen et al., 2004; De Jong and Battjes, 2004; Dong et al., 2013; Dong et al., 2010; Fabrikant, 1995; Gao et al., 2016a; Gao et al., 2017a; Gao et al., 2017b; López and Iglesias, 2013; López et al., 2012; López et al., 2015; Maa et al., 2011; Pattiaratchi and Wijeratne, 2009). Harbor oscillations can have direct influence on the management of ports, shipping and coastal utilization. It has been demonstrated that the management issue most affected by oscillations is harbor operations; the horizontal displacements in the mass of water can induce unacceptable forces on mooring lines and large movements on ships, making moored ship operations much less efficient (López and Iglesias, 2014; Uzaki et al., 2010). As a consequence, the investigation on the water-body oscillations 
inside bays or harbors has a prominent role in the field of coastal engineering.

Among various generation mechanisms, transient harbor oscillations induced by tsunamis are frequently observed and usually destructive. Tsunamis can be triggered by submarine earthquakes, undersea volcanic eruptions or offshore landslides. As tsunamis propagate into the coastal area, the wave height significantly increases owing to continuous decreasing of the water depth (Zhao et al., 2012). For example, the Indian Ocean tsunami, which was induced by the Sumatra earthquake on the December 26, 2004, propagated for about $2 \mathrm{~h}$ to Colombo harbor (Sri Lanka), triggering extreme oscillations with the maximum wave height of $3.87 \mathrm{~m}$ and the resonant period of about 75 min. It then propagated for about $14 \mathrm{~h}$ to Bunbury harbor (Western Australia), triggering oscillations with the maximum wave height of $1.75 \mathrm{~m}$ (Pattiaratchi and Wijeratne, 2009).

So far, studies on transient harbor oscillations induced by tsunamis are mostly confined to using cnoidal waves, solitary waves and $\mathrm{N}$-waves to represent real tsunamis. Using the theoretical and experimental methods, Lepelletier and Raichlen (1987) studied transient nonlinear oscillations inside harbors induced by cnoidal waves. Using laboratory experiments, Dong et al. (2010) explored the resonant response of a rectangular harbor to the tsunamis generated by offshore subaerial landslides. They found that landslide-generated tsunamis usually include components with solitary wave characteristics and also components with dispersive wave characteristics; the former components play an important role in the harbor resonance. Based on a Boussinesq model, they further proved their findings by implementing a series of numerical experiments in which solitary waves were used as the external forcing of the harbor. Subsequently, using a Boussinesq model, Gao et al. (2016b) and Gao et al. (2016c) also carried out a series of numerical experiments on transient harbor resonance triggered by solitary waves, and further analyzed the relative wave energy distribution inside the harbor systematically. Recently, inspired by Tadepalli and Synolakis (1994) who argued that the main tsunami wave was often preceded by a depression and thus introduced the concept of the $\mathrm{N}$-wave in order to achieve better geophysical relevancy, Gao et al. (2017a) employed a fully nonlinear Boussinesq model, FUNWAVE-TVD, to simulate the transient harbor oscillations excited by different types of incident $\mathrm{N}$-waves, and the effects of the incident wave amplitude and the incident $\mathrm{N}$-wave type on the relative wave energy distribution inside the harbor were comprehensively discussed.

Owing to the transformation of tsunamis as they approach the shore, undular bores have been 
often observed in the coastal zone during tsunami events (Grue et al., 2008; Madsen et al., 2008). Madsen et al. (2008) investigated the phenomenon of disintegrated of tsunamis into undular bores, which has been observed, for example, in connection with the Indian Ocean tsunami in 2004, and they also discussed the importance of these undular bores in connection with breaking and runup and concluded that they do no justify a solitary wave model for the bulk tsunami. Peregrine (1966) and, more recently, El et al. (2012) have shown that the evolution of an undular bore on a flat bottom and a gently sloping bottom leads to a system of multi-crested waves which can be viewed as a combination of solitary waves with different wave heights and different separation distances among them. Based on experimental and numerical works, similar findings have also been revealed by Grilli et al. (2012). At present, the investigations on the wave hydrodynamics of successive/multiple solitary waves in the coastal zone are mainly limited on the run-up and back-wash processes over plane slopes. Based on laboratory experiments, Lo et al. (2013) and Xuan et al. (2013) investigated the run-up and back-wash processes of two succussive solitary waves (i.e., double solitary waves) with equal wave heights and various separation distances/times on plane beachs. Using the nonlinear shallow water equations, Dong et al. (2014) studied the evolution and run-up of non-breaking double solitary waves on a plane beach numerically. Subsequently, by using large-scale laboratory experiments, Pujara et al. (2015) studied the interaction of double solitary waves with equal wave heights in the swash zone on a plane beach. Two cases, a strongly interaction case and a weakly interaction one, were condisered. The bed shear stress, bed pressure, and the free-surface displacement at a location near the still-water shoreline were measured to describle how the dynamics of the boundary layer differ when the downrush of the first wave meets the incoming second wave in both cases. Recently, the runup of a train of three successive solitary waves (i.e., triple solitary waves) on a mild slope was investigated experimentally by Rong et al. (2016).

To the best of the authors' knowledge, investigations of transient harbor oscillations induced by a train of successive solitary waves have not been reported in the literature. In order to expand the knowledge of tsunami-induced oscillations, this paper utilizes double solitary waves and triple solitary waves to explore related transient oscillation phenomena. The focus of this paper is to comprehensively investigate the relative wave energy distribution inside the harbor during transient oscillations induced by normal-incident successive solitary waves with different 
amplitudes and different initial incident waveforms. It should be emphasized that it is extremely important to accurately predict and systematically investigate the wave energy distribution inside the harbor excited by tsunamis, which can provide important hydrodynamic information for predicting the motion responses of the moored ships inside the harbor (Kumar et al., 2016; López and Iglesias, 2014). The maximum oscillations (runups) of successive solitary waves inside the harbor are also discussed. Effects of the incident wave height, the relative separation distance between adjacent crests and the number of elementary solitary waves in the incident wave train on these phenomena are systematically investigated. Meanwhile, to compare similarities and differences between oscillations induced by successive solitary waves and those by the single solitary wave, the transient resonance excited by the latter is also simulated, which has been investigated by Gao et al. (2016b) and Gao et al. (2016c) and is only taken as a control group in this paper. Identical to Gao et al. (2017a), all numerical experiments in this article are performed using the fully nonlinear Boussinesq wave model, FUNWAVE-TVD. For simplification, the harbor is assumed to be long and narrow and has a flat bottom; the free-surface movement inside the harbor then essentially becomes one-dimensional.

The rest of the paper is organized as follows. Section 2 briefly describes the numerical model and the wave analysis technique. Section 3 presents the incident wave parameters and the setup of the numerical wave tank. Section 4 demonstrates the simulation results, which are explained in detail. Concluding remarks based on the results are given in Section 5.

\section{Numerical model and data analysis technique}

\subsection{Numerical model}

In this section, the fully nonlinear Boussinesq wave model, FUNWAVE-TVD, which was developed by Shi et al. (2012), is briefly introduced. The model solves the fully nonlinear Boussinesq equations of Chen (2006) using a hybrid finite volume-finite difference scheme, and incorporates a moving reference level as in Kennedy et al. (2001). The equations are reorganized in order to facilitate a high-order shock-capturing TVD (Total Variation Diminishing)-type scheme, which allows the model to be capable of simulating wave breaking and associated wave energy dissipation without relying on empirical formulations (Ma et al., 2012; Shi et al., 2012). Besides, the moving shoreline is captured by a robust wetting-drying scheme, and a third-order Strong Stability-Preserving Runge-Kutta scheme is adopted for adaptive time stepping. The model is 
parallelized using the Message Passing Interface (MPI) with non-blocking communication. With these improvements, the model has been shown to be more robust in predicting wave processes in the nearshore, including wave shoaling, refraction, diffraction, breaking as well as wave runup/rundown on the plane and natural beaches (Shi et al., 2012).

To verify the applicability of FUNWAVE-TVD to the simulation of transient harbor oscillations induced by tsunamis, Gao et al. (2017a) used the numerical model to reproduce the laboratorial and numerical experiments of Dong et al. (2010). Gao et al. (2017a) compared the maximum oscillation inside the harbor predicted by FUNWAVE-TVD with the experimental and numerical data of Dong et al. (2010). It was observed that the numerical results of FUNWAVE-TVD agreed well with both of the experimental data and the numerical results of Dong et al. (2010). It was demonstrated that the numerical model can also simulate transient harbor oscillations excited by tsunamis accurately.

\subsection{Data analysis technique}

The wave conditions inside the harbor for all numerical experiments are analyzed by the normal mode decomposition (NMD) method. The NMD method was proposed originally by Sobey (2006) to calculate the eigenfrequencies, the eigenmodes and the response amplitudes of different modes in natural harbors that are subjected to storm tides or tsunamis. This method consists of two calculating steps. The first step involves the prediction of the discrete set of eigenfrequencies and associated eigenmodes of the semi-enclosed body of water. In the second step, the prediction of the response amplitudes at the discrete resonant modes is formulated and solved as a problem in multi-dimensional optimization, where the eigenfrequencies and eigenmodes calculated in the first step are used as known variables. Recently, the method was improved by Gao et al. (2015) to predict the eigenfrequencies and eigenmodes more accurately. To facilitate the reader's understanding of this paper, the improved NMD method for the one-dimensional harbor is illustrated briefly in this section. For the theory and further details of this method, the interested reader is referred to Sobey (2006) and Gao et al. (2015).

\section{The First Calculating Step}




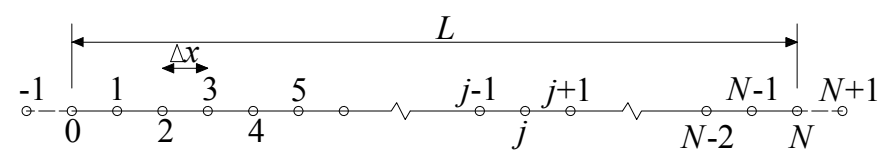

Fig. 1. The one-dimensional uniform grid for closed elongated basins. The actual physical area is from $j=0$ to $j=N$.

For a one-dimensional harbor, the control equation is expressed as

$$
\frac{1}{b} \frac{d}{d x}\left(S \frac{d X}{d x}\right)+\frac{\omega^{2}}{g} X=0
$$

which is a spatially varied Sturm-Liouville equation. The independent variable is the horizontal position $x$. The dependent variable is $X(x ; \omega)$, which is equal to $\eta(x, t) / \cos \omega t . \eta(x, t)$ denotes the local surface elevation with respect to the mean water level; $t$ and $\omega$ denote the time and the angular frequency, respectively. $S(x)$ and $b(x)$ denote the local cross section area and the local surface width. For a uniform grid with node spacing $\Delta x=L / N$ (See Fig. 1. $L$ denotes the harbor length, and $N$ denotes the number of equilong line segments of the harbor length $L$ ), a locally quadratic approximation to Eq. (1) at the interior node $j$ is

$$
a_{j-1} X_{j-1}+a_{j} X_{j}+a_{j+1} X_{j+1}+\frac{\omega^{2}}{g} X_{j}=0
$$

where

$$
a_{j-1}=\frac{S_{j-1}+4 S_{j}-S_{j+1}}{4 b_{j} \Delta x^{2}}, a_{j}=\frac{-2 S_{j}}{b_{j} \Delta x^{2}}, a_{j+1}=\frac{-S_{j-1}+4 S_{j}+S_{j+1}}{4 b_{j} \Delta x^{2}},
$$

and $A_{j}=A(j \Delta x)$ and $b_{j}=b(j \Delta x)$.

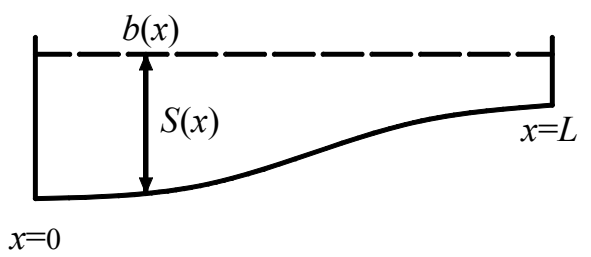

(a)

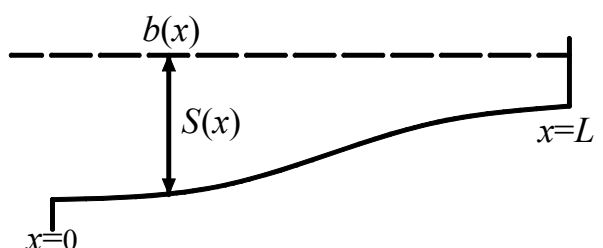

(b)

Fig. 2. Spatially varied closed and partially open elongated basins: (a) closed basin, (b) partially open basin 
For the classical closed-basin problem (see Fig. 2a), discrete approximations of the control equations that are adapted to nodes $j=0$ and $N$ are

$$
a_{0} X_{0}+a_{1}^{*} X_{1}+\frac{\omega^{2}}{g} X_{0}=0
$$

and

$$
a_{N-1}^{*} X_{N-1}+a_{N} X_{N}+\frac{\omega^{2}}{g} X_{N}=0
$$

respectively, where

$$
\begin{aligned}
& a_{0}=\frac{-2 S_{0}}{b_{0} \Delta x^{2}}, a_{1}^{*}=\frac{2 S_{0}}{b_{0} \Delta x^{2}} \\
& a_{N}=\frac{-2 S_{N}}{b_{N} \Delta x^{2}}, a_{N-1}^{*}=\frac{2 S_{N}}{b_{N} \Delta x^{2}} .
\end{aligned}
$$

Defining the column vector $\mathbf{X}=\left(X_{0}, X_{1}, X_{2} \ldots X_{N-1}, X_{N}\right)^{\mathrm{T}}$, Eq. (2) for nodes 1 through $N-1$, together with Eqs. (4) and (5) at nodes 0 and $N$, can be written

$$
\mathbf{A X}=\lambda \mathbf{X}
$$

where $\mathbf{A}$ is a tridiagonal matrix of $a_{j}$ coefficients, with eigenvalue $\lambda=-\omega^{2} / g$. For a partially open basin (see Fig. $2 \mathrm{~b}$ ), $X_{0}=0$ so that the column vector $\mathbf{X}$ is $\left(X_{1}, X_{2} \ldots X_{N-1}, X_{N}\right)^{\mathrm{T}}$. Both the row and column dimensions of matrix A become $N$, Eq. (2) defining rows 1 through $N-1$, with Eq. (5) for row $N$. Further, the eigenfrequencies of the harbor, $\omega_{n}$, can be calculated from the eigenvalue $\lambda$, and the eigenvectors of the matrix $\mathbf{A}$ are the eigenmodes of the harbor, $\mathbf{X}_{n}$.

For the one-dimensional harbor problem, the following normalization condition for the eigenmodes

$$
\left.\mathbf{X}_{n}\right|_{j=N}=1
$$

is adopted.

\section{The Second Calculating Step}

For the one-dimensional harbor, the response amplitudes are calculated using the following fitting equation

$$
\eta(x, t)=\sum_{n}\left[a_{1 n} \cos \omega_{n} t+a_{2 n} \sin \omega_{n} t\right] \mathbf{X}_{n}(x)
$$

The response amplitudes $a_{1 n}, a_{2 n}$ would then be available as an optimization problem. The suitable objective function to be minimized would be

$$
S\left(a_{1 n}, a_{2 n}\right)=\sum_{i k}\left[\eta_{i k}-\sum_{n=1}^{M}\left(a_{1 n} \cos \omega_{n} t_{k}+a_{2 n} \sin \omega_{n} t_{k}\right) \mathbf{X}_{n}\left(x_{i}\right)\right]^{2},
$$


where $M$ denotes the number of modes of which the response amplitudes should be decomposed. The eigenmode amplitudes of different resonant modes are defined as

$$
A_{n}=\left[a_{1 n}^{2}+a_{2 n}^{2}\right]^{1 / 2} .
$$

Subsequently, by using the NMD method, Gao et al. (2016b) and Gao et al. (2016c) systematically analyzed the relative wave energy distribution inside the harbor during transient harbor oscillations induced by single solitary waves with different heights. Similar to Gao et al. (2016b) and Gao et al. (2016c), all numerical experiments in this paper also adopt this method to analyze the relative wave energy distribution inside the harbor induced by different types of incident tsunamis.

It should be noted that in the second step, the fitting function, Eq. (9), indicates that the free surfaces inside the harbor are linearly superposed by all resonant modes, and the nonlinear interactions of different modes are not considered in this equation. Therefore, in theory, the fitting function is only valid for the linear wave condition inside the harbor. In fact, Gao et al. (2015) and Gao et al. (2016c) have proved that this fitting function can also calculate accurately the response amplitudes of the different modes for weakly nonlinear wave condition inside the harbor; while for the wave condition inside the harbor with stronger nonlinearity, the calculating results would become inaccurate and unreliable. Therefore, in this paper, the incident tsunamis used in all numerical experiments are only confined to the linear waves.

\section{Numerical experimental setup}

\subsection{Incident wave parameters}



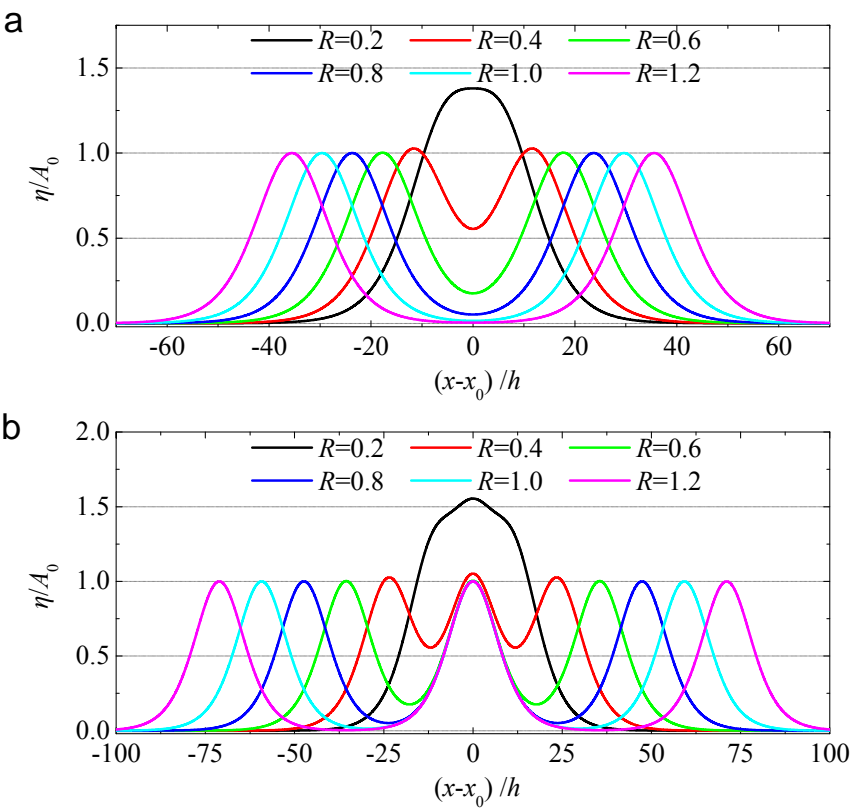

Fig. 3. Wave profiles of (a) double solitary waves and (b) triple solitary waves with $R$ ranging from 0.2 to 1.2 with an increment of 0.2 under the conditions of $A_{0} / h=0.015$ and $h=10.0 \mathrm{~m}$.

For a single solitary wave which travels in the $x$-direction in a constant depth at the speed of $c$, the leading-order solution is well-known:

$$
\eta(x, t)=A_{0} \operatorname{sech}^{2}[k(x-c t)], \text { where } k=\sqrt{\frac{3 A_{0}}{4 h^{3}}} \text {, and } c=\sqrt{g\left(A_{0}+h\right)},
$$

and

$$
L=\frac{2 \pi}{k}, \text { and } T=\frac{2 \pi}{k c},
$$

can be viewed as the effective wavelength and effective wave period, respectively. $A_{0}$ and $h$ denotes the wave height of the single solitary wave and water depth, respectively.

In this paper, the double and the triple solitary waves are generated by the linear superposition of two and three elementary solitary waves with equal wave heights and various separation distances between adjacent crests. Hence, the initial free-surface elevations in numerical experiments for single solitary wave (SSW), double solitary waves (DSW) and triple solitary waves (TSW) are expressed as

$$
\eta(x)=\left\{\begin{array}{l}
A_{0} \operatorname{sech}^{2}\left[k\left(x-x_{0}\right)\right], \text { for SSW } \\
A_{0}\left[\operatorname{sech}^{2}\left(k\left(x-x_{0}-R \cdot L / 2\right)\right)+\operatorname{sech}^{2}\left(k\left(x-x_{0}+R \cdot L / 2\right)\right)\right], \text { for DSW } \\
A_{0}\left[\operatorname{sech}^{2}\left(k\left(x-x_{0}-R \cdot L\right)\right)+\operatorname{sech}^{2}\left(k\left(x-x_{0}\right)\right)+\operatorname{sech}^{2}\left(k\left(x-x_{0}+R \cdot L\right)\right)\right], \text { for TSW }
\end{array}\right.
$$

and the initial velocity is taken as a linear expression 


$$
u(x)=\sqrt{\frac{g}{h}} \eta(x),
$$

in which $x_{0}$ denotes the center position of the initial incident wave train, and $R$ denotes the relative separation distance between adjacent crests, which is defined as the ratio of the distance between adjacent crests in the incident wave train to the effective wavelength of the SSW, $L$.

For all numerical experiments studied in this article, the water depth at the open ocean is set to a constant, $h=10.0 \mathrm{~m}$ (this will be described in detail in Section 3.2). To intuitively demonstrate the waveform of the DSW and TSW with various relative separation distances, Fig. 3 shows the wave profiles of DSW and TSW with $R$ ranging from 0.2 to 1.2 with an increment of 0.2 under the conditions of $A_{0} / h=0.015$ and $h=10.0 \mathrm{~m}$. For the DSW (Fig. 3a), when $R$ equals to 0.2 , the two elementary solitary waves merge into one single-peak wave. When $R$ increases to 0.4 , the maximum free-surface elevation of the DSW, $\eta_{\mathrm{m}}$, is slightly larger than the wave height of the elementary solitary wave, $A_{0}$, that is, $\eta_{\mathrm{m}}=1.03 A_{0}$. When $R$ becomes equal to or larger than 0.6 , the maximum free-surface elevation of the DSW becomes nearly equal to $A_{0}$. For the TSW (Fig. 3b), the similar phenomena can be also observed. When $R$ equals to 0.2 , the three elementary solitary waves merge into one single-peak wave. When $R$ increase to 0.4 , the maximum free-surface elevation of the TSW is also slightly larger than $A_{0}$, that is, $\eta_{\mathrm{m}}=1.05 A_{0}$. When $R$ becomes equal to or larger than 0.6 , the maximum free-surface elevation of the TSW becomes nearly equal to $A_{0}$. Hence, in this paper, only the successive solitary waves with $R \geq 0.4$ will be investigated.

Table 1 presents the specific parameters of different types of initial incident waves for all of the simulations. This paper includes five groups of numerical experiments, namely, Groups A-E. To systematically investigate the effect of the wave height variation on the relative wave energy distribution inside the harbor, the dimensionless wave height of the elementary solitary wave, $A_{0} / h$, in these groups ranges from 0.005 to 0.025 . Each of the five groups consists of nine cases with different types of initial incident waves, which include the SSW, the DSW with $R$ ranging from 0.4 to 1.2 in an increment of 0.2 and the TSW with $R=0.4,0.6$ and 0.8 . It should be noted that the related transient oscillation phenomena induced by the SSW, including the wave energy distribution and the maximum wave oscillation (runup) inside the harbor, has been investigated in Gao et al. (2016b) and Gao et al. (2016c). The purpose of considering the SSW is to understand which features are common and which are different regarding these phenomena excited by the 
successive solitary waves and by the SSW. Fig. 4 compares the waveforms of the various types of initial incident waves with $A_{0} / h=0.015$ and $R=0.4$ and 0.8 . The open circle in the figure refers to the maximum free-surface elevation of the corresponding waveform, and the solid one stands for the corresponding wavefront, which is defined as $\eta_{\mathrm{f}}=0.05 \eta_{\mathrm{m}}$, where $\eta_{\mathrm{f}}$ denotes the free-surface elevation of the wavefront. As has been shown in Fig. 3, for the case of $R=0.4$ (Fig. 4a), both the maximum free-surface elevations of the DSW and the TSW are slightly larger than that of the SSW, by approximately 1.03 and 1.05 times, respectively; while for the case of $R=0.8$ (Fig. 4b), the three types of initial incident waves have almost identical maximum free-surface elevation. As expected, for the same value of $R$, the length scale of the TSW is significantly larger than that of the DSW, and meanwhile the latter is notably larger than that of the SSW.

a

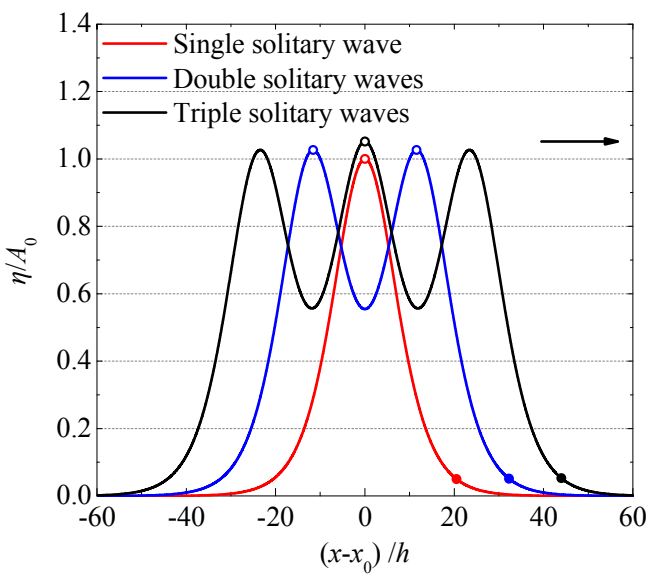

b

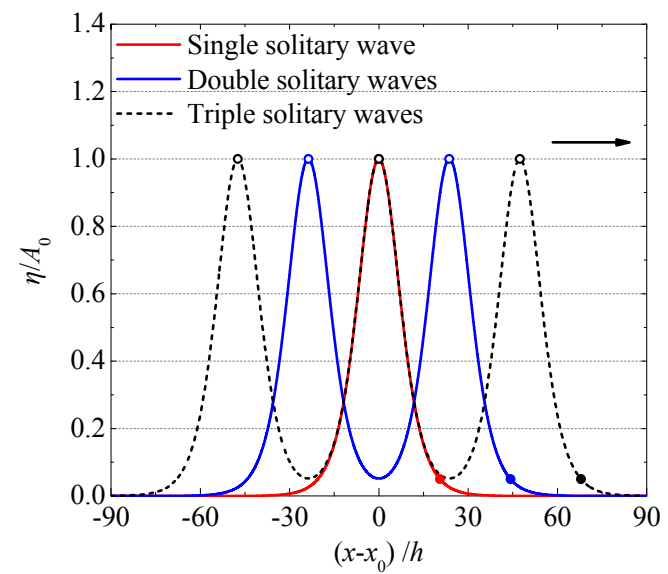

Fig. 4. Waveform comparison of the different initial incident waves for (a) $R=0.4$ and (b) $R=0.8$ under the conditions of $A_{0} / h=0.015$ and $h=10.0 \mathrm{~m}$. The open and solid circles denote the maximum free-surface elevation and the wavefront of the corresponding waveform, respectively. 
Table 1. Wave parameters of the initial incident waves in all numerical experiments

\begin{tabular}{|c|c|c|c|c|c|}
\hline Group & $A_{0} / h$ & Initial incident waveform & Case & $R$ & $\eta_{\mathrm{m}} / A_{0}$ \\
\hline \multirow{9}{*}{ A } & \multirow{9}{*}{0.005} & SSW & A01 & -- & 1.00 \\
\hline & & \multirow{5}{*}{ DSW } & A02 & 0.4 & 1.03 \\
\hline & & & $\mathrm{A} 03$ & 0.6 & 1.00 \\
\hline & & & A04 & 0.8 & 1.00 \\
\hline & & & A05 & 1.0 & 1.00 \\
\hline & & & A06 & 1.2 & 1.00 \\
\hline & & \multirow{3}{*}{ TSW } & A07 & 0.4 & 1.05 \\
\hline & & & A08 & 0.6 & 1.00 \\
\hline & & & A09 & 0.8 & 1.00 \\
\hline \multirow{9}{*}{ B } & \multirow{9}{*}{0.010} & SSW & B01 & -- & 1.00 \\
\hline & & \multirow{5}{*}{ DSW } & B02 & 0.4 & 1.03 \\
\hline & & & B03 & 0.6 & 1.00 \\
\hline & & & B04 & 0.8 & 1.00 \\
\hline & & & B05 & 1.0 & 1.00 \\
\hline & & & B06 & 1.2 & 1.00 \\
\hline & & \multirow{3}{*}{ TSW } & B07 & 0.4 & 1.05 \\
\hline & & & B08 & 0.6 & 1.00 \\
\hline & & & B09 & 0.8 & 1.00 \\
\hline \multirow{9}{*}{$\mathrm{C}$} & \multirow{9}{*}{0.015} & SSW & $\mathrm{C} 01$ & -- & 1.00 \\
\hline & & \multirow{5}{*}{ DSW } & $\mathrm{C} 02$ & 0.4 & 1.03 \\
\hline & & & $\mathrm{C} 03$ & 0.6 & 1.00 \\
\hline & & & $\mathrm{C} 04$ & 0.8 & 1.00 \\
\hline & & & $\mathrm{C} 05$ & 1.0 & 1.00 \\
\hline & & & $\mathrm{C} 06$ & 1.2 & 1.00 \\
\hline & & \multirow{3}{*}{ TSW } & $\mathrm{C} 07$ & 0.4 & 1.05 \\
\hline & & & C08 & 0.6 & 1.00 \\
\hline & & & C09 & 0.8 & 1.00 \\
\hline \multirow{9}{*}{$\mathrm{D}$} & \multirow{9}{*}{0.020} & SSW & D01 & -- & 1.00 \\
\hline & & \multirow{5}{*}{ DSW } & D02 & 0.4 & 1.03 \\
\hline & & & D03 & 0.6 & 1.00 \\
\hline & & & D04 & 0.8 & 1.00 \\
\hline & & & D05 & 1.0 & 1.00 \\
\hline & & & D06 & 1.2 & 1.00 \\
\hline & & \multirow{3}{*}{ TSW } & D07 & 0.4 & 1.05 \\
\hline & & & D08 & 0.6 & 1.00 \\
\hline & & & D09 & 0.8 & 1.00 \\
\hline \multirow{9}{*}{ E } & \multirow{9}{*}{0.025} & SSW & E01 & -- & 1.00 \\
\hline & & \multirow{5}{*}{ DSW } & E02 & 0.4 & 1.03 \\
\hline & & & E03 & 0.6 & 1.00 \\
\hline & & & E04 & 0.8 & 1.00 \\
\hline & & & E05 & 1.0 & 1.00 \\
\hline & & & E06 & 1.2 & 1.00 \\
\hline & & \multirow{3}{*}{ TSW } & E07 & 0.4 & 1.05 \\
\hline & & & E08 & 0.6 & 1.00 \\
\hline & & & E09 & 0.8 & 1.00 \\
\hline
\end{tabular}




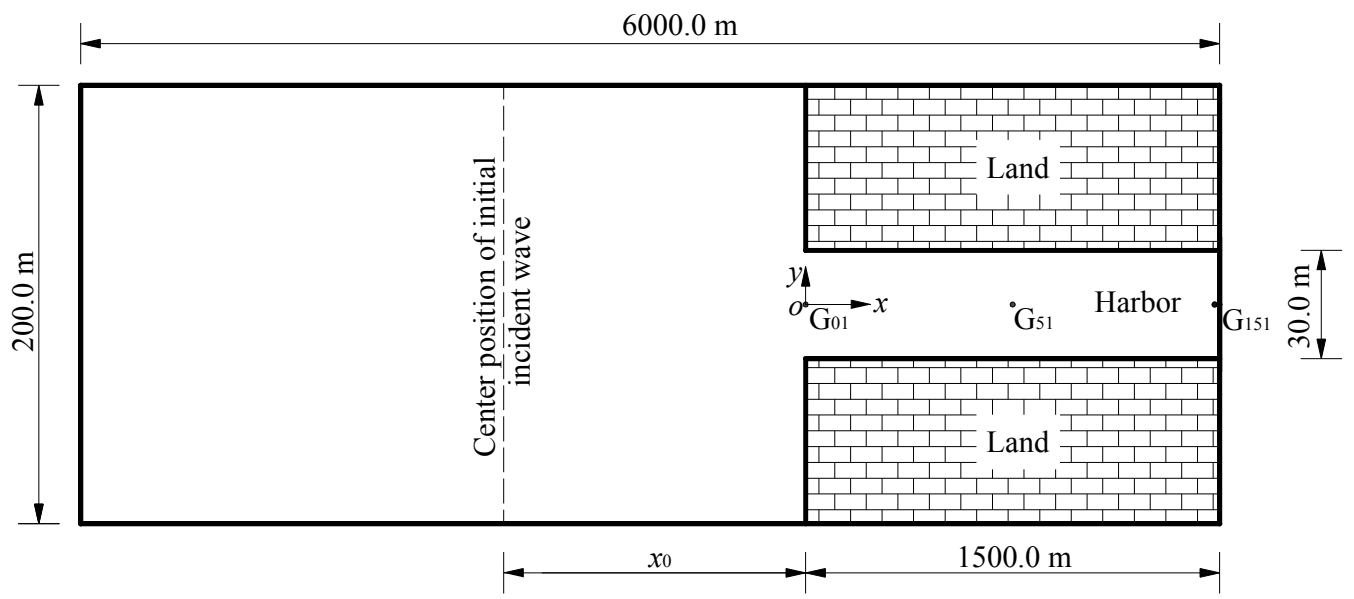

Fig. 5. Sketch of the numerical experimental setup used in the numerical experiments. Based on its geometrical symmetry with respect to the $x$-axis, only half of the domain (i.e., $y \geq 0$ ) is used as the computational domain for simulations.

Fig. 5 illustrates the sketch of the numerical experimental setup used in the numerical experiments. The dimensions of the numerical wave tank are $6000.0 \mathrm{~m} \times 200.0 \mathrm{~m}$. A long and narrow rectangular harbor with constant depth is adopted. The harbor has a length of $1500.0 \mathrm{~m}$ and a width of $30.0 \mathrm{~m}$. The water depth inside and outside the harbor is set to $h=10.0 \mathrm{~m}$. To reduce the computational cost, only half of the domain (i.e., $y \geq 0$ ) is used for simulations based on the geometrical symmetry with respect to the $x$-axis. There are 151 wave gauges $\left(\mathrm{G}_{01}-\mathrm{G}_{151}\right)$ arranged equidistantly with a spacing of $10.0 \mathrm{~m}$ along the center line of the harbor. Gauges $\mathrm{G}_{01}$ and $\mathrm{G}_{151}$ are those at the entrance and the backwall of the harbor, respectively. The grid sizes $\Delta x$ and $\Delta y$ are uniform in $1.0 \mathrm{~m}$ throughout the numerical wave tank, and the computational domain (i.e., $y \geq 0$ ) consists of 606,101 nodes and 600,000 rectangular elements. All of the boundaries are set to be fully reflective. As an initial condition, in each case, the wavefront of the initial incident waves is set at the harbor entrance. The simulation time is $500.0 \mathrm{~s}$ for all cases.

To examine whether the grid size $\Delta x=\Delta y=1.0 \mathrm{~m}$ is fine enough to obtain convergent simulation results, two other different grid sizes, i.e., $\Delta x=\Delta y=0.5 \mathrm{~m}$ and $\Delta x=\Delta y=2.0 \mathrm{~m}$, are also utilized to reproduce the numerical simulations for cases C01, C04 and C09. The size and layout of the numerical wave tank and the initial incident wave condition for the three different grid sizes are set to be identical. Fig. 6 compares the time series of the wave elevations at gauge $\mathrm{G}_{151}$ for the 
three different grid sizes for all the three cases. It can be observed that the simulation results obtained by the three different grid sizes are almost identical to each other, which indicates that the grid size $\Delta x=\Delta y=1.0 \mathrm{~m}$ adopted in this paper is able to obtain convergent simulation results.
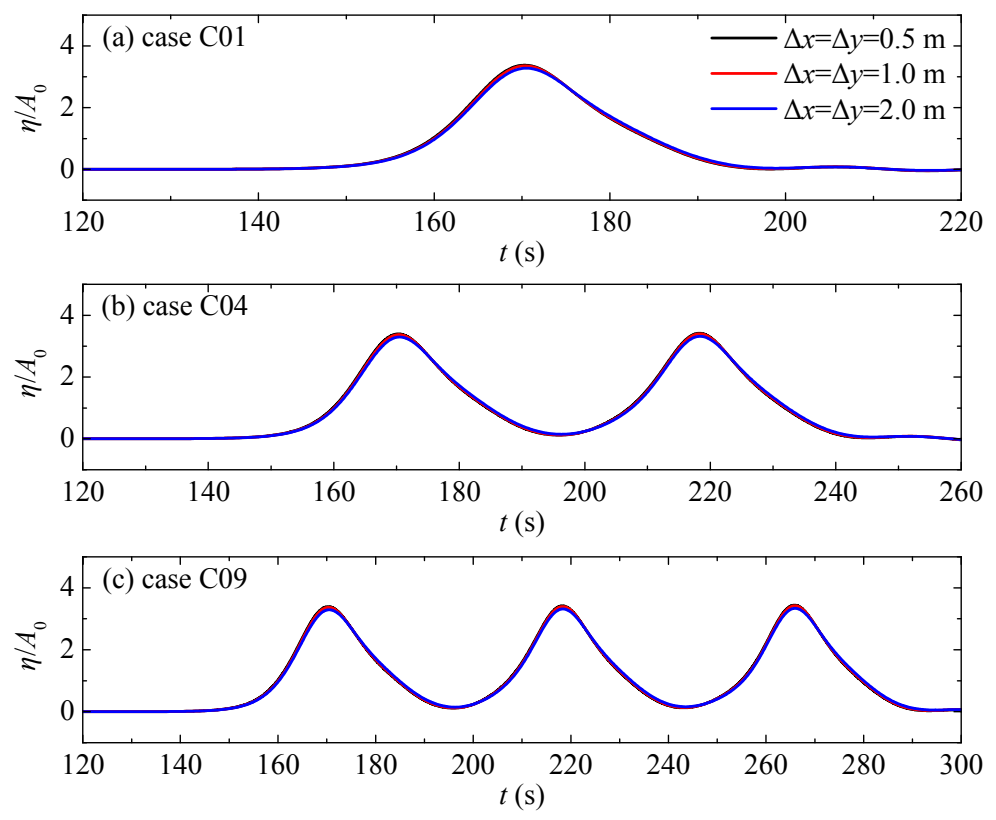

Fig. 6. Time series of the wave elevations at gauge $\mathrm{G}_{151}$ for different grid sizes for cases $\mathrm{C} 01, \mathrm{C} 04$ and $\mathrm{C} 09$.

\section{Results and discussion}

4.1. Calculation process of the response amplitude of the different modes

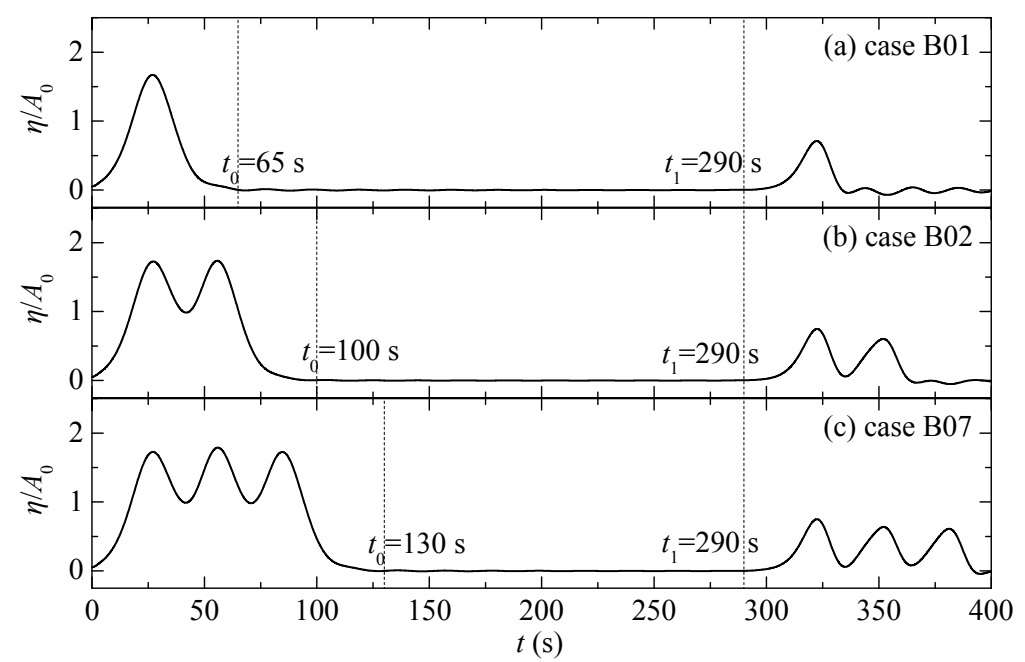

Fig. 7. Time series of the wave elevations at gauge $\mathrm{G}_{01}$ for cases $\mathrm{B} 01, \mathrm{~B} 02$ and $\mathrm{B} 07$

In all numerical experiments, only the wave conditions during the period from $t_{0}$ to $t_{1}$ are used to separate the response amplitudes of the different resonant modes inside the harbor. $t_{0}$ and $t_{1}$ 
denote the moment that the incident wave completely enters the harbor from the open sea and the moment that the reflected wave from the backwall of the harbor begins to propagate out of the harbor, respectively. Take cases B01, B02 and B07 for example. Fig. 7 shows the time series of the wave elevations at gauge $\mathrm{G}_{01}$ in these cases. For case B01 (Fig. 7a), the incident SSW completely enters the harbor at $t_{0}=65.0 \mathrm{~s}$, and the reflected wave from the backwall begins to propagate out of the harbor at $t_{1}=290.0 \mathrm{~s}$. For case B02 in which the DSW is used as the initial incident wave (Fig. $7 \mathrm{~b}$ ), the value of $t_{0}$ increases to $100.0 \mathrm{~s}$, and the value of $t_{1}$ keeps the same with that in case B01. For case B07 in which the TSW is used as the initial incident wave (Fig. 7c), the value of $t_{0}$ increases further to $130.0 \mathrm{~s}$, and the value of $t_{1}$ still remains the same with that in case B01.

a
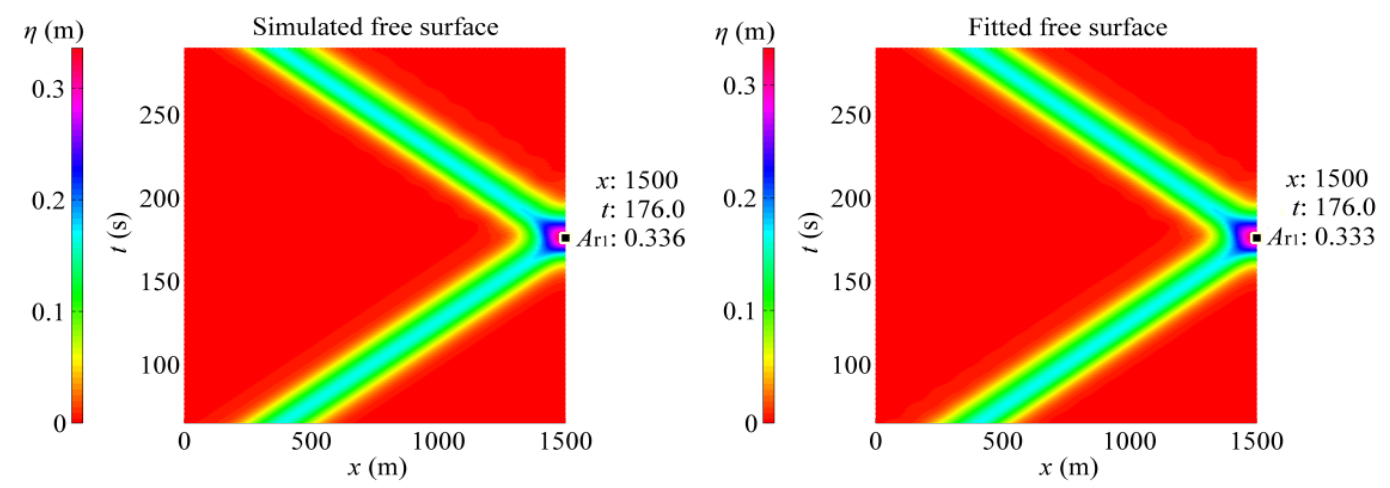

b
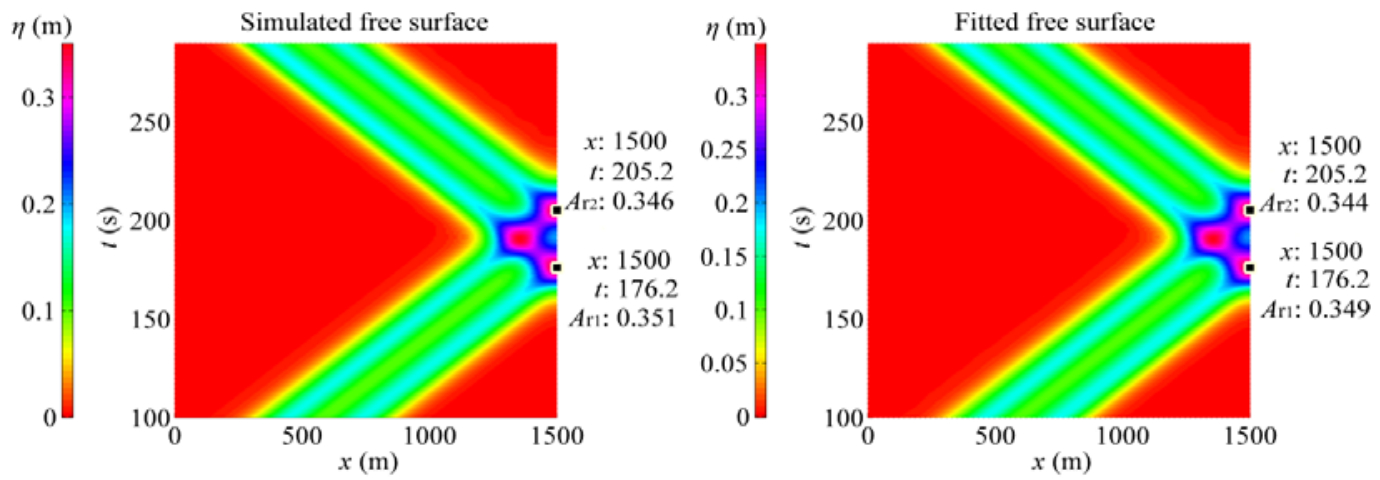

C
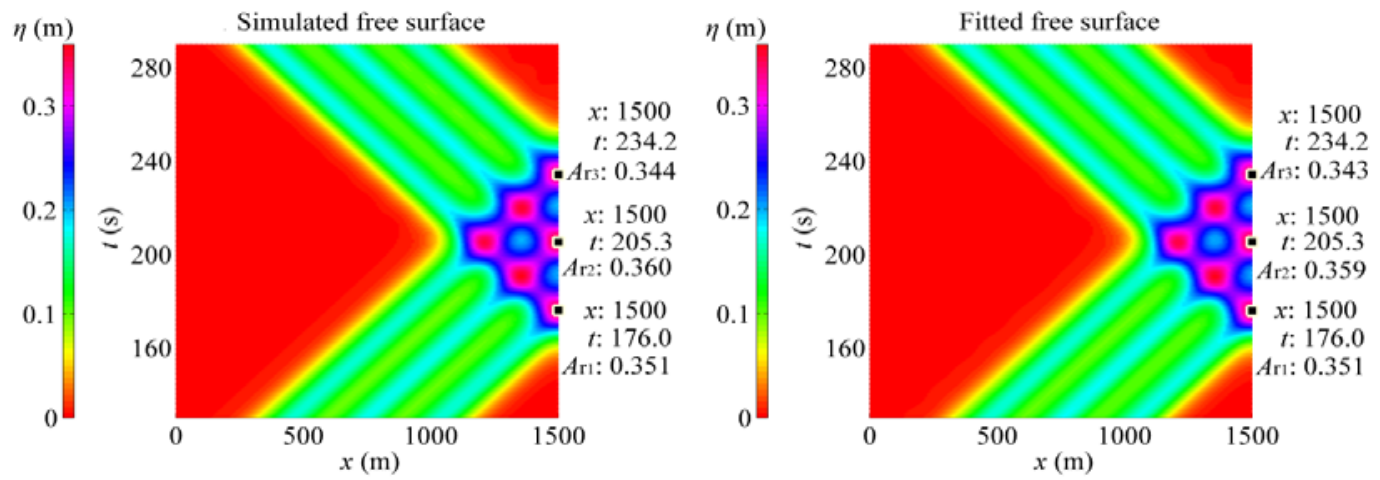

Fig. 8. Comparison of the simulated free surfaces and the corresponding fitted free surfaces for (a) case B01, (b) case B02 and (c) case B07 
Fig. 8 shows the comparison of the simulated free surfaces using the FUNWAVE-TVD model and the corresponding fitted free surfaces using the NMD method in cases B01, B02 and B07. The lowest 40 resonant modes are considered in fitting the free surfaces. For all the three cases, the fitted free surfaces coincide very well with the simulated ones. For case B01 (Fig. 8a), the simulated free surface inside the harbor has a maximum runup of $0.336 \mathrm{~m}$ at $x=1500.0 \mathrm{~m}$ and $t=176.0 \mathrm{~s}$; at the same position and the same time, the fitted free surface has a maximum runup of 0.333 m. For case B02 (Fig. 8b), the simulated free surface inside the harbor has two maximum runups at $x=1500.0 \mathrm{~m}$. The first maximum runup has a value of $0.351 \mathrm{~m}$ at $t=176.2 \mathrm{~s}$, and the second one has a value of $0.346 \mathrm{~m}$ at $t=205.2 \mathrm{~s}$. At the same position and the same time, the fitted free surface has the first maximum runup of $0.349 \mathrm{~m}$ and the second maximum runup of $0.344 \mathrm{~m}$. For case B07 (Fig. 8c), the simulated free surface inside the harbor has three maximum runups at $x=1500.0 \mathrm{~m}$. The three maximum runups have values of $0.351 \mathrm{~m}, 0.360 \mathrm{~m}$ and $0.344 \mathrm{~m}$ occurring at $t=176.0 \mathrm{~s}, 205.3 \mathrm{~s}$ and $234.2 \mathrm{~s}$, respectively. At the same position and the same time, the fitted free surface also has three maximum runups, and their corresponding values are $0.351 \mathrm{~m}, 0.359 \mathrm{~m}$ and $0.343 \mathrm{~m}$. Owing to the significance and typicality of these maximum runups, we define the numerical fitting error $(N F E)$ of the NMD method to be the maximum relative error between the simulated and the fitted maximum runups, that is,

$$
N F E=\left\{\begin{array}{l}
\frac{\left|\left(A_{\mathrm{r} 1}\right)_{\text {fitted }}-A_{\mathrm{r} 1}\right|}{A_{\mathrm{r} 1}} \times 100 \%, \text { for SSW } \\
\max \left\{\frac{\left|\left(A_{\mathrm{r} 1}\right)_{\text {fitted }}-A_{\mathrm{r} 1}\right|}{A_{\mathrm{r} 1}}, \frac{\left|\left(A_{\mathrm{r} 2}\right)_{\mathrm{fitted}}-A_{\mathrm{r} 2}\right|}{A_{\mathrm{r} 2}}\right\} \times 100 \%, \text { for DSW } \\
\max \left\{\frac{\left|\left(A_{\mathrm{r} 1}\right)_{\text {fitted }}-A_{\mathrm{r} 1}\right|}{A_{\mathrm{r} 1}}, \frac{\left|\left(A_{\mathrm{r} 2}\right)_{\mathrm{fitted}}-A_{\mathrm{r} 2}\right|}{A_{\mathrm{r} 2}}, \frac{\left(A_{\mathrm{r} 3}\right)_{\text {fitted }}-A_{\mathrm{r} 3} \mid}{A_{\mathrm{r} 3}}\right\} \times 100 \%, \text { for TSW }
\end{array}\right.
$$

in which $A_{\mathrm{ri}}(i=1,2,3)$ denotes the simulated $i^{\text {th }}$ maximum runup, and $\left(A_{\mathrm{r} i}\right)_{\text {fitted }}$ denotes the fitted $i^{\text {th }}$ maximum runup. The NFE reflects the accuracy of the NMD method in separating the response amplitudes of the different resonant modes. The NFEs for cases B01, B02 and B07 are 0.89\%, $0.58 \%$ and $0.38 \%$, respectively. Fig. 9 demonstrates the NFEs in all numerical experiments. It can be found that, overall, the NFE for each type of the initial incident waveform increases with the incident wave height, which agrees with the finding in Gao et al. (2015). The NFEs in all numerical experiments are less than $5.0 \%$, which guarantees the accurate prediction of the 
response amplitudes of different resonant modes in all simulations.

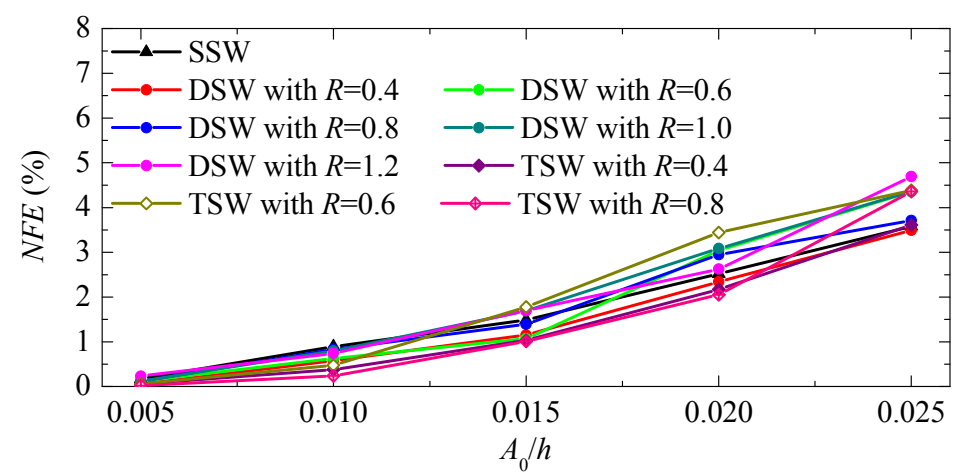

Fig. 9. Numerical fitting errors (NFEs) for all cases

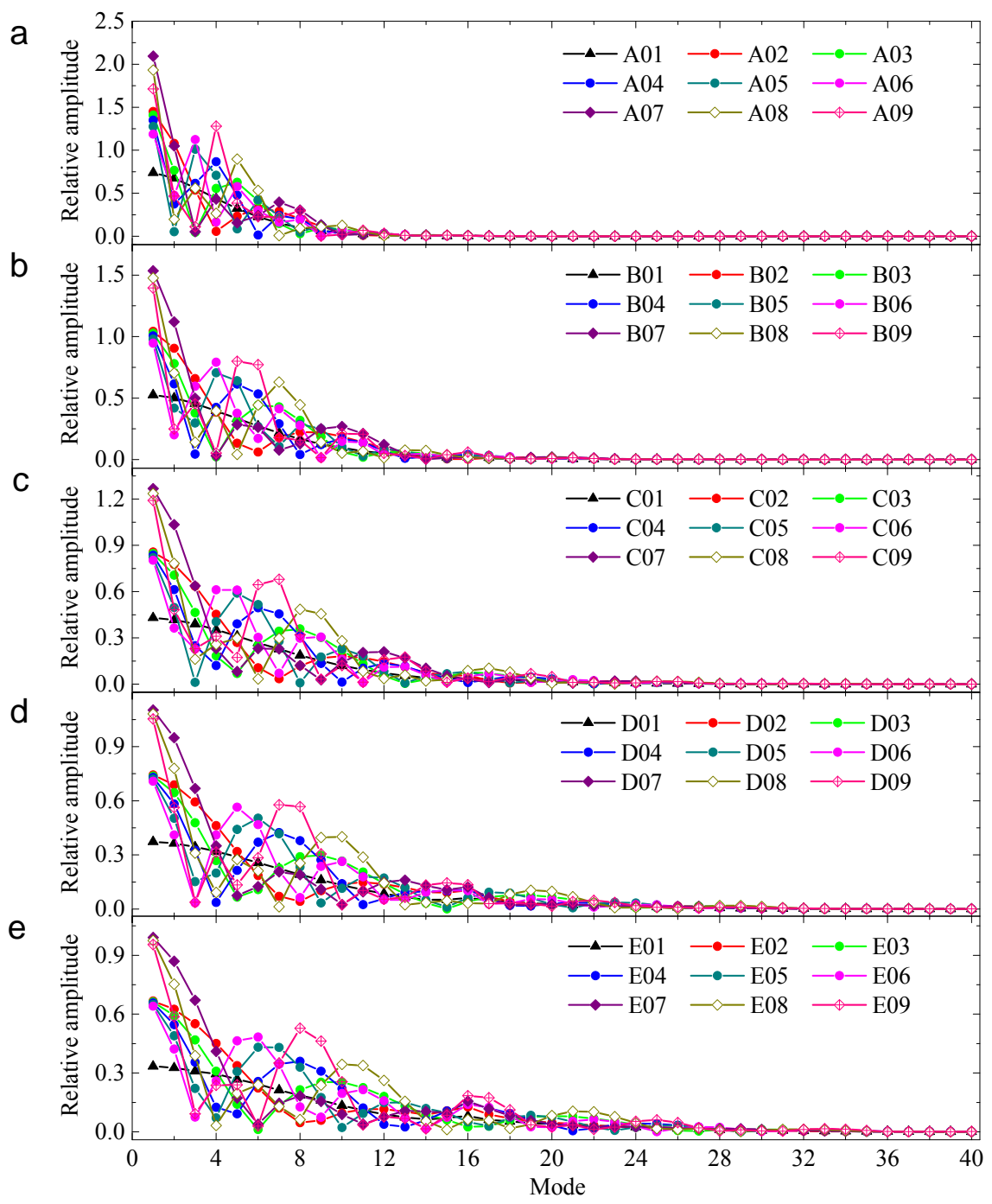

Fig. 10. Relative amplitude distributions of the lowest 40 resonant modes for all the cases in (a)

Group A, (b) Group B, (c) Group C, (d) Group D and (e) Group E 
In this paper, the "relative amplitude" in each case is defined as the ratio of the response amplitude of each resonant mode to the wave height of the elementary solitary wave, $A_{0}$, that is,

$$
\bar{A}_{i}=\frac{A_{i}}{A_{0}} \quad(i=1,2, \ldots, 40)
$$

Fig. 10 illustrates the relative amplitude distributions of the lowest 40 resonant modes in all numerical experiments. From the figure, it is easily observed that all the following three parameters, that is, the incident wave height, the relative separation distance between adjacent crests and the number of elementary solitary waves in the incident wave train, have dramatic influences on the relative wave energy distributions inside the harbor. The concrete influences of these three parameters on the relative wave energy distribution inside the harbor will be elaborated and analyzed in Sections $4.2-4.4$, respectively.

\subsection{Effect of the incident wave height on the relative wave energy}

Fig. 11 illustrates the variations of the relative amplitude distributions with respect to the wave height of the elementary solitary wave, $A_{0}$, for the SSW, the DSW and the TSW with $R=0.4$. For all the three types of the initial incident waves, it can be easily observed that, when the incident wave height is small, the wave energy inside the harbor is dominated by the lowest few resonant modes, and only a small proportion of the wave energy is distributed over the higher modes. However, with the increase of the incident wave height, most of the wave energy inside the harbor is distributed over more resonant modes. In general, the relative energy distribution inside the harbor tends to become more uniform for the larger incident wave. In addition, for all the three types of the initial incident waves, in the variation range of $A_{0}$ studied in this paper, the lowest resonant mode always possesses the highest wave energy inside the harbor, which can be observed more comprehensively in Fig. 10. This phenomenon is quite different from the corresponding finding in Gao et al. (2017a) about the transient harbor oscillations excited by the N-waves. Gao et al. (2017a) found that for various types of incident $\mathrm{N}$-waves, the resonant mode that possesses the highest wave energy shifts gradually from a lower mode to the higher mode with the increase of the incident wave amplitude. 


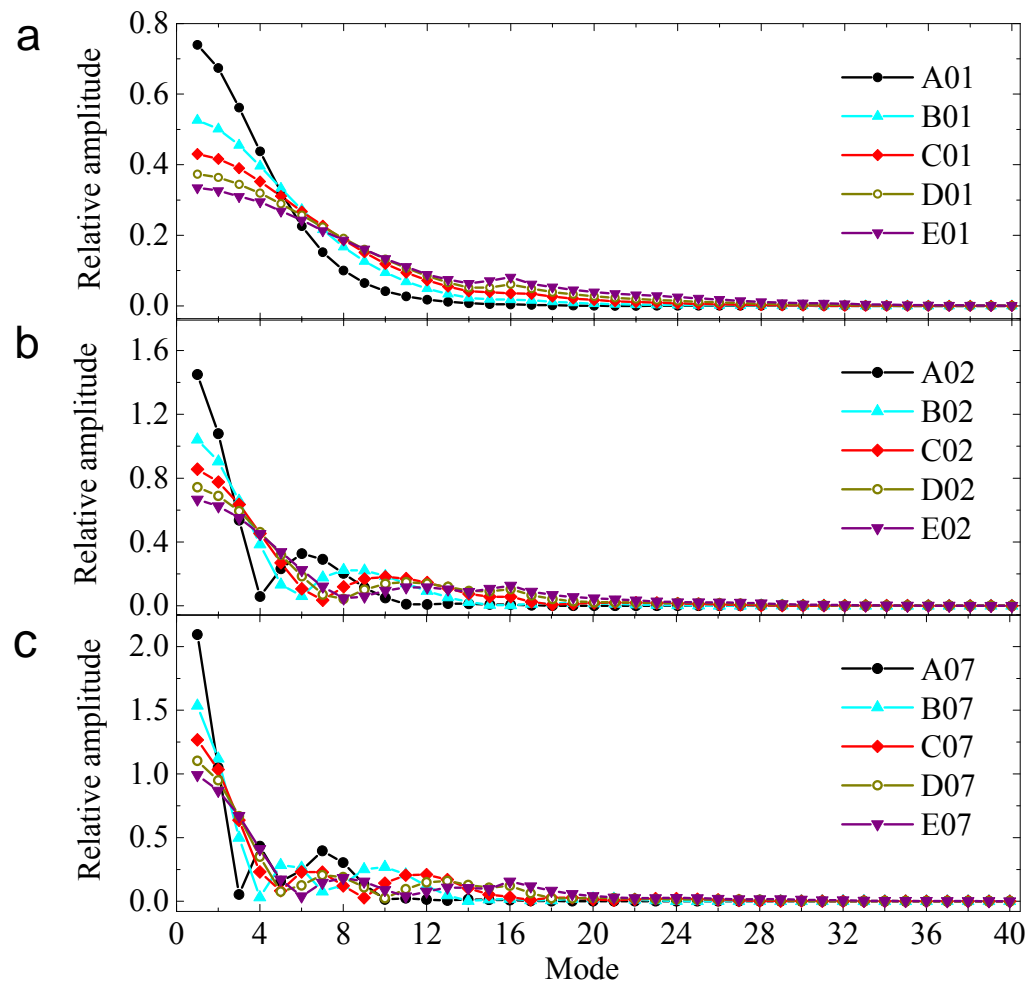

Fig. 11. Variations of the relative amplitude distributions with respect to the wave height of the elementary solitary wave, $A_{0}$, for (a) the SSW, (b) the DSW with $R=0.4$ and (c) the TSW with $R=0.4$

Identical to Gao et al. (2017a), to qualitatively compare the uniformity of the relative wave energy distribution inside the harbor, the coefficients of variance of the relative amplitude distribution over the lowest 40 modes for all cases are calculated. The coefficient of variance $(C V)$ is defined as

$$
C V=\frac{\sigma}{\mu},
$$

in which

$$
\begin{gathered}
\sigma=\sqrt{\frac{1}{40} \sum_{i=1}^{40}\left(\bar{A}_{i}-\mu\right)^{2}}, \\
\mu=\frac{1}{40} \sum_{i=1}^{40} \bar{A}_{i} .
\end{gathered}
$$

It can intuitively reflect the discrete degree of the relative wave amplitude of different resonant modes relative to their average value. Obviously, the smaller the coefficient of variance is, the more uniform the wave energy distribution inside the harbor over different modes is. Fig. 12 illustrates the variations of the $C V$ values of the relative amplitude distributions with respect to the 
dimensionless wave height of the elementary solitary wave, $A_{0} / h$, for the SSW, the DSW and the TSW with various relative separation distances between adjacent crests. It is clearly shown that for all the three types of the incident waves, the $C V$ value decreases monotonically with the increase of the incident wave height, which is consistent with the qualitative findings for Fig. 11.

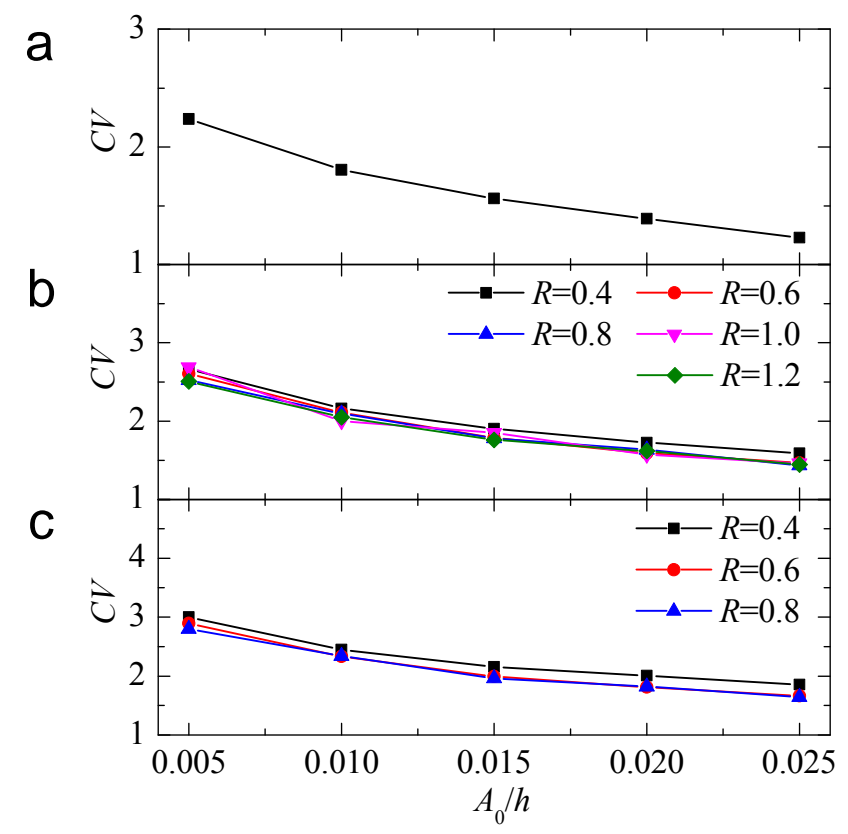

Fig. 12. The $C V$ values of the relative amplitude distributions inside the harbor for (a) the SSW, (b) the DSW and (c) the TSW

4.3. Effect of the relative separation distance between adjacent crests on the relative wave energy
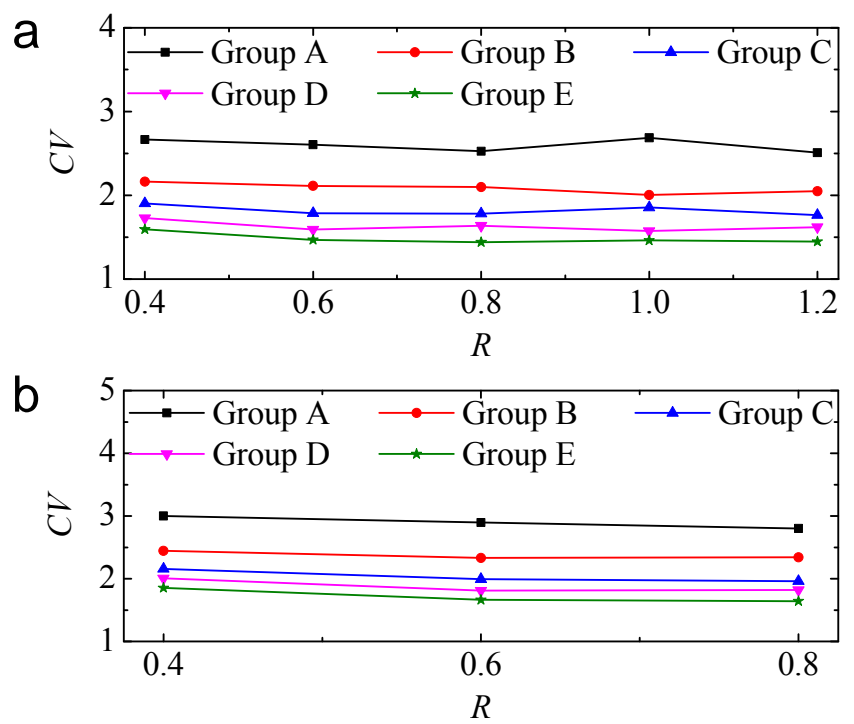

Fig. 13. Variations of the $C V$ values with respect to the relative separation distance between adjacent crests, $R$, for (a) the DSW and (b) TSW in Groups A-E 
In order to reveal the effect of the relative separation distance between adjacent crests in the successive solitary wave train on the uniformity of the wave energy distribution inside the harbor, Fig. 13 illustrates the variations of the $C V$ values of the relative amplitude distributions with respect to the relative separation distance, $R$, for the DSW and the TSW in all groups. From this figure, it is seen that for both the DSW and the TSW, the $C V$ value seems to be insensitive to $R$ in its variation range studied in this paper. Take cases A02-A06 and cases A07-A09, for example. For cases A02-A06 (see Group A in Fig. 13a), the average $C V$ value for the five cases is equal to 2.59, while the standard deviation of the five $C V$ values is only 0.08 . Similarly, for cases A07-A09 (see Group A in Fig. 13b), the average $C V$ value for the three cases is equal to 2.90, while their standard deviation is only 0.09 . Hence, it is indicated from this figure that, in the variation range of $R$ considered in this paper, the relative separation distance between adjacent crests in the successive solitary wave train has a negligible influence on the overall uniformity of the relative wave energy distribution inside the harbor.

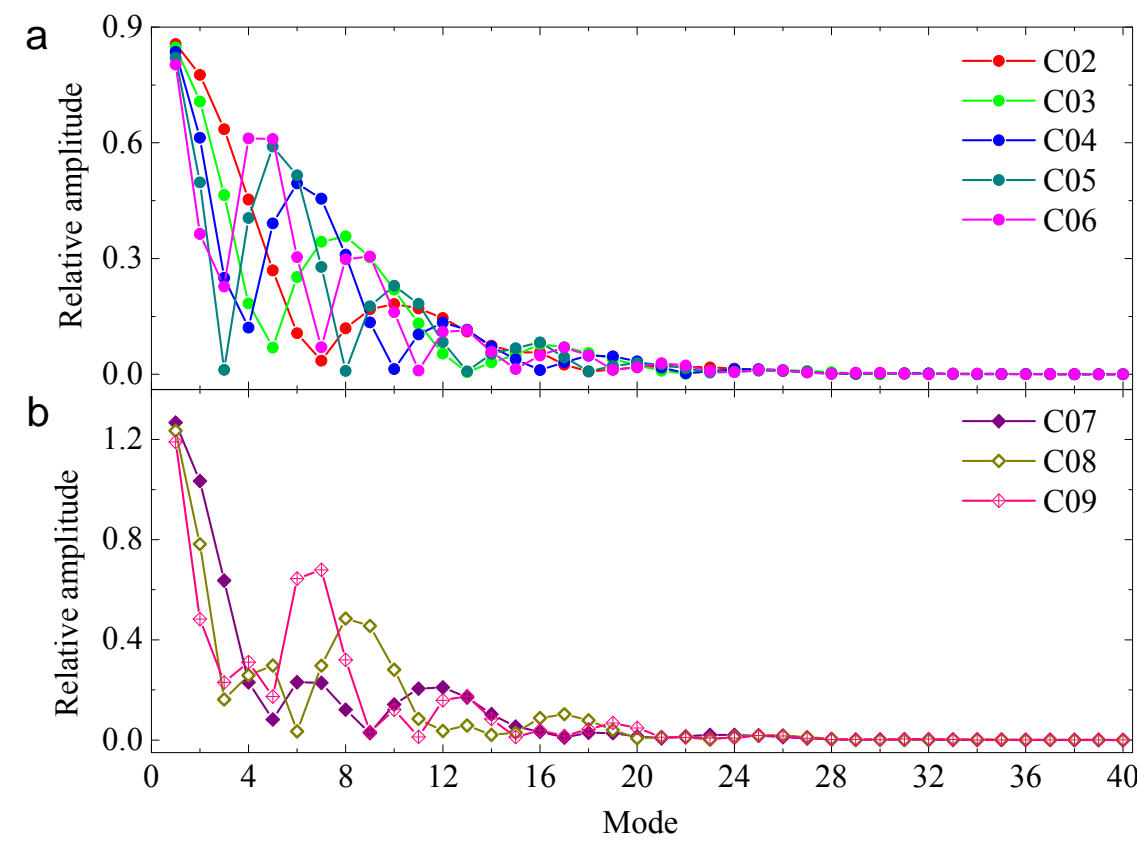

Fig. 14. Relative amplitude distributions of the lowest 40 resonant modes for (a) the DSW and (b) the TSW in Group C

By further observing Fig. 10, it can be found that only the one parameter (i.e. the $C V$ value) cannot comprehensively reflect the effects of the relative separation distance between adjacent 
crests in the successive solitary wave train on the wave energy distribution inside the harbor. It can be intuitively seen from Fig. 10 that for both the DSW and the TSW in all groups, a larger relative separation distance between adjacent crests causes more obvious fluctuations of the relative amplitude distribution inside the harbor. To show this phenomenon more clearly, the relative amplitude distribution for the DSW and the TSW in Group C are illustrated separately in Fig. 14. For the DSW (Fig. 14a), when the value of $R$ equals to 0.4 (case C02), there only exist two summits in the relative amplitude distribution, that is, the relative amplitudes on the $1^{\text {st }}$ and the $10^{\text {th }}$ modes. As the relative separation distance between adjacent crests increases, the number of summits in the relative amplitude distribution gradually increases. When the value of $R$ increases to 1.2 (case C06), there exist up to six summits in the relative amplitude distribution, that is, the relative amplitudes on the $1^{\text {st }}$, the $4^{\text {th }}$, the $9^{\text {th }}$, the $13^{\text {th }}$, the $17^{\text {th }}$ and the $21^{\text {st }}$ modes. For the TSW (Fig. 14b), the similar phenomenon can be also observed. To demonstrate this phenomenon more comprehensively, Fig. 15 further presents the number of summits in the relative wave amplitude distributions, $N_{\mathrm{S}}$, for the DSW and the TSW in all groups. It is clearly shown that, for both the DSW and TSW in all the five groups, the number of summits in the relative wave amplitude distribution always increases gradually with the relative separation distance between adjacent crests, $R$.
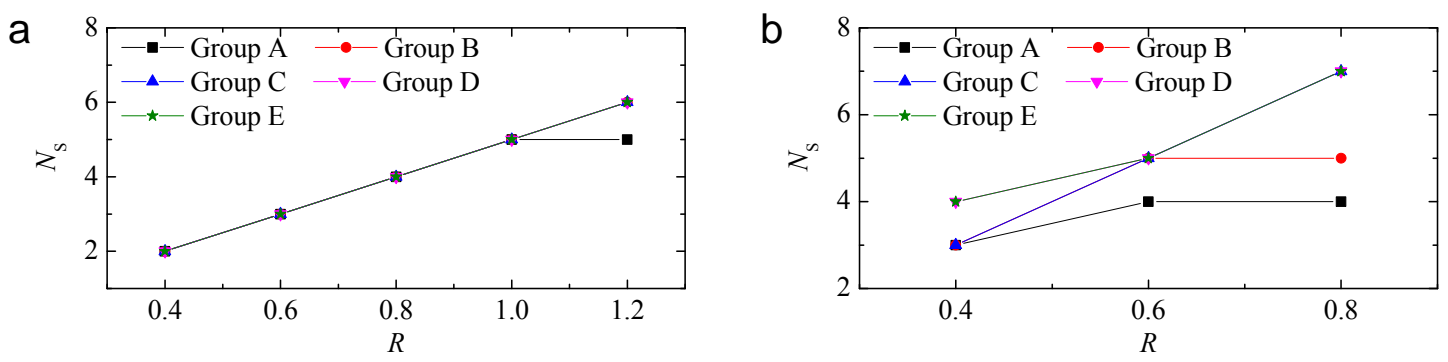

Fig. 15. The number of summits in the relative wave amplitude distributions, $N_{\mathrm{S}}$, for (a) the DSW and (b) the TSW in Groups A-E

Observing Fig. 10 can also find that in each case, the lowest resonant mode always possesses the highest relative wave amplitude, no matter whether the incident wave train is the SSW, the DSW or the TSW. Therefore, it can be speculated that the lowest resonant mode is normally the most dangerous for the ships moored inside the harbor, and should be paid more attention on. The 
sensitivity of the response amplitude of lowest resonant modes with respect to the relative separation distance between adjacent crests in the successive solitary wave train is further analyzed. Fig. 16 illustrates the variations of the response amplitudes of the lowest mode with respect to the relative separation distance, $R$, for the DSW and the TSW in all groups. For the DSW (Fig. 16a), when the incident wave height is small in Group A, the response amplitude of the lowest mode remarkably decreases with the relative separation distance, $R$. The value of $A_{1} / A_{0}$ decreases from 1.45 in case A02 to 1.19 in case A06, which falls by up to $17.9 \%$. However, as the incident wave height increases, although the response amplitude of the lowest mode still decreases with the relative separation distance between adjacent crests, its descent trend becomes weaker. In Group E, the value of $A_{1} / A_{0}$ decreases from 0.66 in case E02 to 0.64 in case E06, which falls only by $3.0 \%$. For the TSW (Fig. 16b), the same phenomenon can be also observed. When the incident wave height is small in Group A, the value of $A_{1} / A_{0}$ decreases from 2.09 in case $\mathrm{A} 07$ to 1.71 in case A09, which falls by up to $18.2 \%$. While in Group E, the value of $A_{1} / A_{0}$ decreases from 0.99 in case E07 to 0.95 in case E09, which falls only by $4.0 \%$.
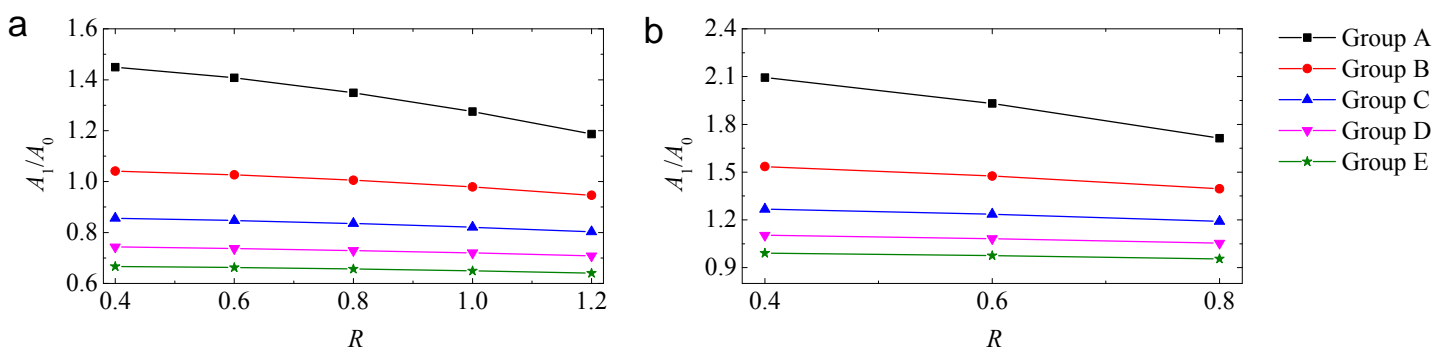

Fig. 16. Variations of the response amplitudes of the lowest mode with respect to the relative separation distance between adjacent crests, $R$, for (a) the DSW and (b) the TSW in Groups A-E

4.4. Effect of the number of elementary solitary waves in the wave train on the relative wave energy

From Fig. 10, it can be also observed that the relative amplitude distribution inside the harbor induced by different types of the incident tsunamis (i.e., the SSW, the DSW and the TSW) presents some different characteristics. In other words, the number of elementary solitary waves in the wave train has certain obvious influences on the relative wave energy distribution inside the harbor, which are mainly reflected in the following two asepcts. First, in each group, the larger the 
number of elementary solitary waves in the incident wave train is, the more uniform the relative amplitude distribution over the lowest 40 modes becomes. Second, the larger number of elementary solitary waves in the wave train tends to cause the more notable fluctuation of the relative amplitude distribution inside the harbor. These two distinct differences will be described in detail in the next two paragraphs, respectively.

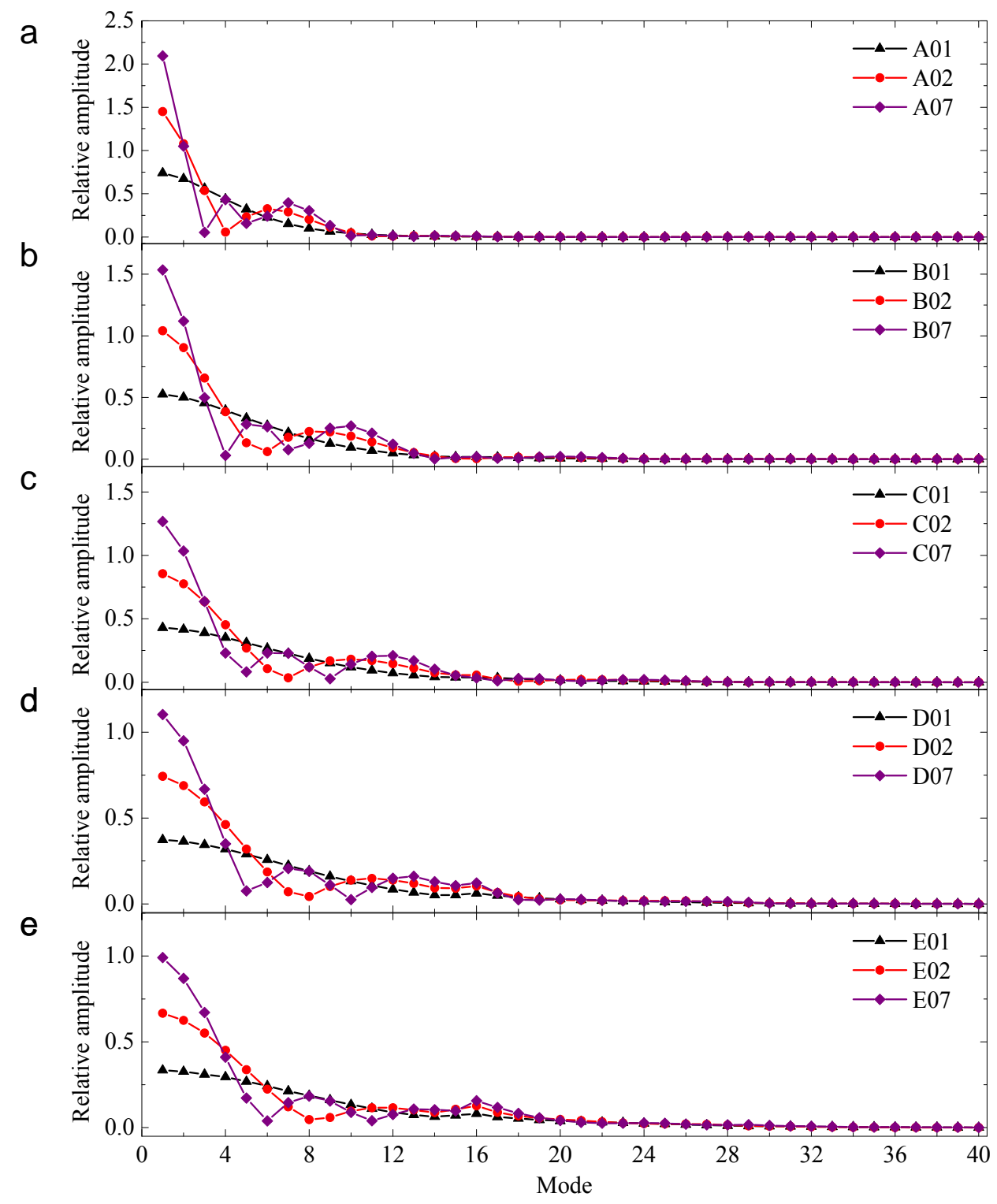

Fig. 17. Comparison of the relative amplitude distribution for the SSW, the DSW and the TSW with $R=0.4$ in (a) Group A, (b) Group B, (c) Group C, (d) Group D and (e) Group E

To show the influence of the number of elementary solitary waves in the wave train on the relative wave energy inside the harbor more clearly, Fig. 17 illustrates the comparison of the 
relative amplitude distribution for the SSW, the DSW and the TSW with $R=0.4$ in Groups A-E. In each group, the highest relative amplitude in the lowest mode induced by the TSW is always remarkably larger than that excited by the DSW; meanwhile, the latter is always considerably greater than that induced by the SSW. Take the three cases in Group A (Fig. 17a), for example. For case A07 in which the TSW is used as the initial incident wave, the highest relative amplitude in the lowest mode equals to 2.09 , and then the relative amplitude decreases sharply as the resonant mode increases. The wave energy inside the harbor is mainly concentrated in the lowest two modes. For case A02 in which the DSW is used as the initial incident wave, the highest relative amplitude in the lowest mode decreases to 1.45 , and the wave energy inside the harbor is mainly concentrated in the lowest three modes. For case A01 in which the SSW is used as the initial incident wave, the highest relative amplitude in the lowest mode decreases further to 0.74 . Compared to cases A07 and A02, as the resonant mode increases, the decay rate of the wave energy becomes much milder, and the wave energy inside the harbor is mainly concentrated in the lowest eight modes. To describe this phenomenon more comprehensively, the variations of the $C V$ values with respect to the number of elementary solitary waves in the wave train, $N$, in Groups AE are illustrated in Fig. 18. It is found that in all the five groups, the value of $C V$ always increases monotonically with $N$ for all the three $R$ values, which coincides with the phenomenon shown in Figs. 10 and 17.
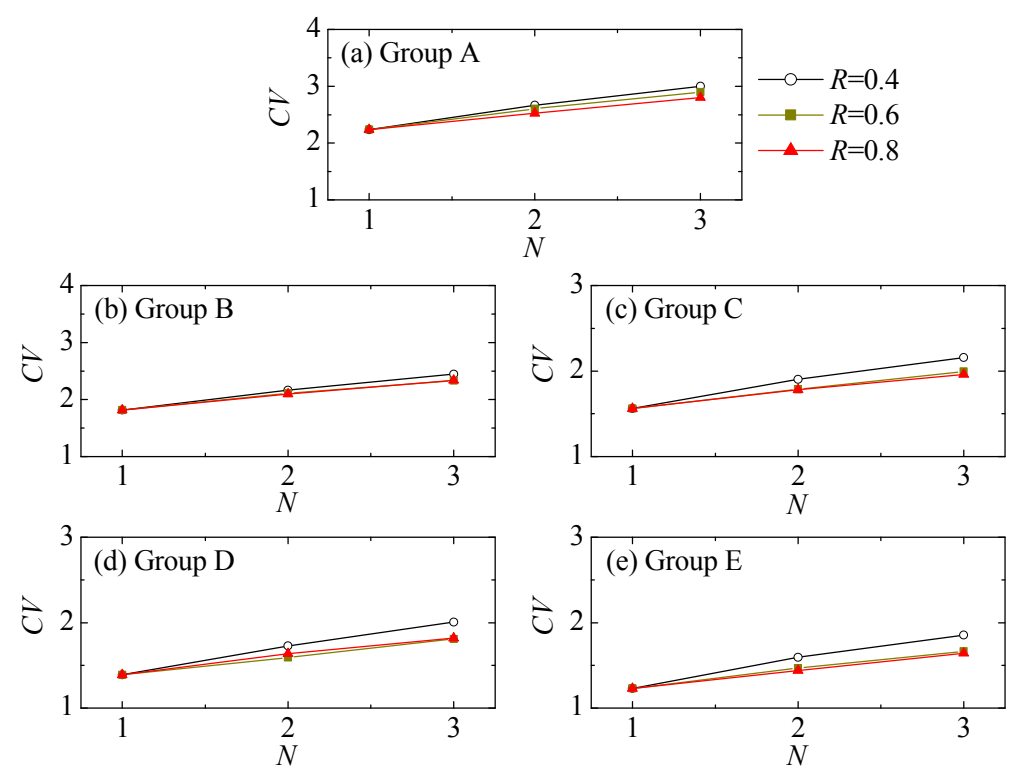

Fig. 18. Variations of the $C V$ values with respect to the number of elementary solitary waves in 
the wave train, $N$, in Groups A-E
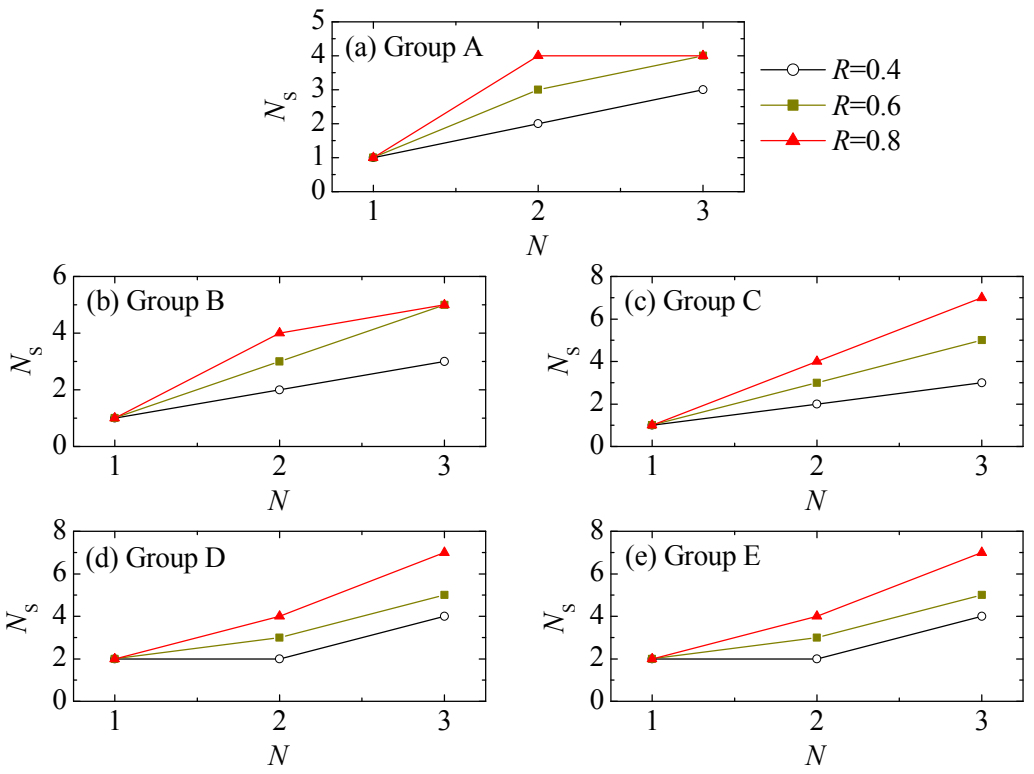

Fig. 19. Variations of the number of summits in the relative amplitude distribution, $N_{\mathrm{S}}$, with respect to the number of elementary solitary waves in the wave train, $N$, in Groups A-E

By observing Fig. 17, it can be also clearly seen that in each group, for a certain value of $R$, the larger number of elementary solitary waves in the wave train tends to cause the more notable fluctuation of the relative amplitude distribution inside the harbor, which is reflected in the number of summits in the relative amplitude distribution. Take the three cases in Group A (Fig. 17a), for example again. For case A01, there is only one summit in the relative amplitude distribution, that is, the relative amplitude on the lowest mode. For case A02, the number of summits in the relative amplitude distribution increases to two, which occur on the $1^{\text {st }}$ and the $6^{\text {th }}$ modes. For case A07, the number of summits in the relative amplitude distribution increases further to three, which appear on the $1^{\text {st }}$, the $4^{\text {th }}$ and the $7^{\text {th }}$ modes. To show the phenomenon more comprehensively, Fig. 19 illustrates the variations of the number of summits in the relative amplitude distribution with respect to the number of elementary solitary waves in the wave train in Groups A-E. It can be easily found that in all the five groups, the number of summits in the relative amplitude distribution tends to increase with the number of elementary solitary waves in the wave train for all the three $R$ values. 


\subsection{Maximum oscillations inside the harbor}

To study the relationship between the wave height of the incident SSW and the maximum oscillation (runup) inside the harbor, Dong et al. (2010), Gao et al. (2016b) and Gao et al. (2016c) implemented a series of numerical experiments and found that the maximum oscillation (runup) inside the harbor with flat or constant-slope bottom grows linearly with the wave height of the incident SSW when the wave nonlinearity inside the harbor is relatively weak. In the current study, this discussion is extended to the maximum oscillation of the DSW and the TSW inside the harbor.
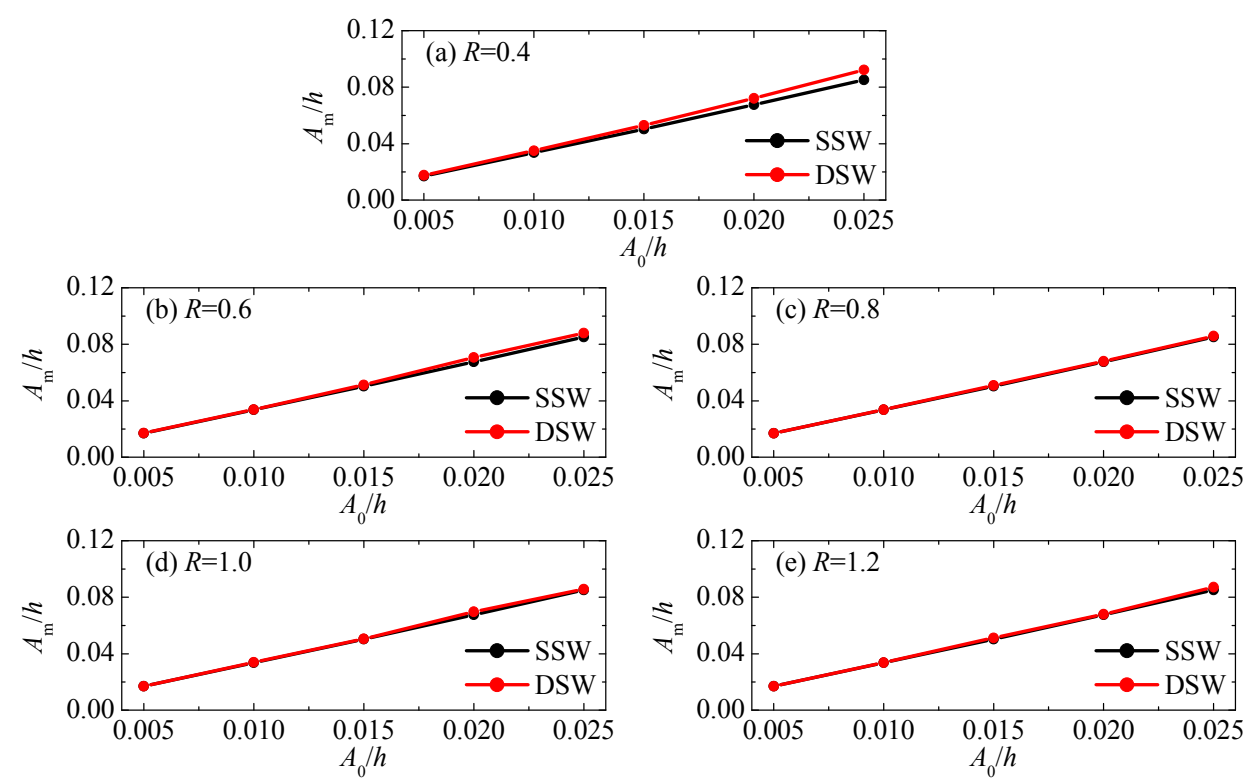

Fig. 20. Comparison of the maximum oscillation inside the harbor induced by the SSW and the DSW with various relative separation distances between adjacent crests, $R$

Different from the SSW that only induces one maximum runup inside the harbor, the DSW and the TSW generate two and three maximum runups inside the harbor, respectively (refer to Fig. 8). Hence, the maximum oscillation inside the harbor in each case is defined as

$$
A_{\mathrm{m}}=\left\{\begin{array}{l}
A_{\mathrm{r} 1}, \text { for the SSW } \\
\max \left(A_{\mathrm{r} 1}, A_{\mathrm{r} 2}\right), \text { for the DSW } \\
\max \left(A_{\mathrm{r} 1}, A_{\mathrm{r} 2}, A_{\mathrm{r} 3}\right), \text { for the TSW }
\end{array}\right.
$$

Fig. 20 illustrates the comparison of the maximum oscillation inside the harbor induced by the SSW and the DSW with various relative separation distances between adjacent crests, $R$. When the relative separation distance $R=0.4$ (Fig. 20a), the maximum oscillation induced by the DSW is 
slightly larger than that induced by the SSW, which is due to that the maximum free-surface elevation of the initial incident DSW, $\eta_{\mathrm{m}}$, is slightly larger than the wave height of the initial incident SSW, $A_{0}$ (refer to Fig. 4 and Table 1). However, when $R$ becomes equal to or greater than 0.6 (Fig. 20b-e), the maximum free-surface elevation of the initial incident DSW, $\eta_{\mathrm{m}}$, becomes nearly equal to the wave height of the initial incident SSW, $A_{0}$. The maximum oscillation inside the harbor induced by the former becomes almost identical to that excited by the latter. Similar to Fig. 20, Fig. 21 shows the comparison of the maximum oscillation inside the harbor induced by the SSW and the TSW with different relative separation distances between adjacent crests, $R$. When $R$ equals to 0.4 (Fig. 21a), the maximum oscillation induced by the TSW is slightly larger than that induced by the SSW due to the same reason; when $R$ becomes equal to or greater than 0.6 (Fig. $21 \mathrm{~b}$ and c), the maximum oscillation inside the harbor induced by the TSW becomes almost identical to that excited by the SSW.
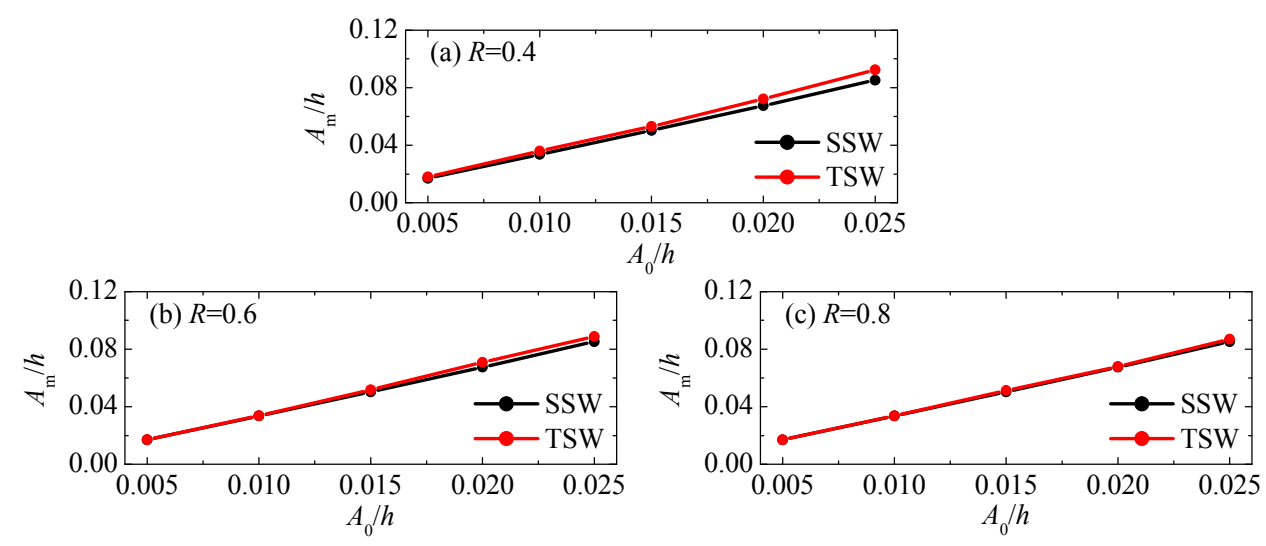

Fig. 21. Comparison of the maximum oscillation inside the harbor induced by the SSW and the TSW with different relative separation distances between adjacent crests, $R$

\section{Conclusions}

Due to the transformation of tsunamis as they approach the shore, undular bores have been often observed in the coastal zone during tsunami events (Grue et al., 2008; Madsen et al., 2008). Meanwhile, the evolution of an undular bore on a flat bottom and a gently sloping bottom leads to a system of multi-crested waves which can be viewed as a combination of solitary waves (El et al., 2012; Peregrine, 1966). Based on the above-mentioned phenomena, in this paper, the transient oscillations inside an elongated rectangular harbor excited by succussive solitary waves (i.e., the 
double and the triple solitary waves) with different incident wave heights and various relative separation distances between adjacent crests are first simulated using the FUNWAVE-TVD model. To reveal the similarities and differences between the resonance induced by the successive solitary waves and that excited by the single solitary wave, the latter is also simulated in the current paper, acting as a control group. Based on the numerical simulations and the NMD method (Gao et al., 2015; Sobey, 2006), this article focuses on the influences of the incident wave height, the relative separation distance between adjacent crests and the number of elementary solitary waves in the incident wave train on the relative wave energy distribution inside the harbor. The maximum oscillation inside the harbor excited by the various types of the incident waves has also been studied. The results of this study will improve the understanding of transient harbor oscillations induced by tsunamis.

The following conclusions can be drawn from the results of the present investigation:

1. The relative wave energy distribution inside the harbor is greatly influenced by the incident wave height. For all the three types of the incident tsunamis considered in this paper (i.e., the SSW, the DSW and the TSW), when the incident wave height is small, the vast majority of the wave energy is concentrated in the lowest few resonant modes. As the incident wave height increases, the proportion of the wave energy distributed over the higher modes increases. In conclusion, the initial incident tsunami with a larger wave height leads to more uniform relative wave energy distribution inside the harbor.

2. For the successive solitary waves (i.e., the DSW and the TSW), the relative separation distance between adjacent crests also has dramatic influences on the relative wave energy distribution inside the harbor, and they are reflected in the following two aspects. First, although the relative separation distance between adjacent crests has a negligible influence on the overall uniformity of the relative wave energy distribution inside the harbor, a larger relative separation distance between adjacent crests leads to more obvious fluctuations of the relative amplitude distribution inside the harbor. Second, when the incident wave height is small, the maximum response amplitude inside the harbor, which is always possessed by the lowest mode, is also sensitive to the relative separation distance between adjacent crests. The maximum response amplitude inside the harbor decreases significantly with the increase of the relative separation distance between adjacent crests. 
3. Besides the incident wave height and the relative separation distance between adjacent crests, the relative wave energy distribution inside the harbor is also greatly affected by the number of elementary solitary waves in the incident wave train, which is also reflected in two aspects. First, the initial incident tsunami with more elementary solitary waves leads to more uniform relative wave energy distribution inside the harbor. Second, the larger number of elementary solitary waves in the incident tsunami tends to cause more notable fluctuations of the relative amplitude distribution inside the harbor.

4. When the wave height of the elementary solitary wave in the successive solitary waves equals to that of the SSW and the relative separation distance between adjacent crests is less than 0.6 , the maximum oscillation inside the harbor induced by the successive solitary waves is slightly larger than that excited by the SSW. However, when the relative separation distance between adjacent crests further increases, the maximum oscillation induced by the former becomes almost identical to that excited by the latter.

Finally, we reaffirm here that these conclusions are only valid for the elongated harbor with constant depth and when the relative height of the elementary solitary wave, $A_{0} / h$, is less than 0.025 and the relative separation distance between adjacent crests, $R$, is at the range of 0.4-1.2.

\section{Acknowledgments}

This work was financially supported by the National Natural Science Foundation of China (Grant nos. 51609108, 51309124 and 51679113), the Natural Science Foundation of the Jiangsu Higher Education Institutions of China (Grant no. 16KJB570004), the State Key Laboratory of Coastal and Offshore Engineering at Dalian University of Technology (Grant no. LP1602), the Jiangsu Key Laboratory of Coast Ocean Resources Development and Environment Security at Hohai University (Grant no. JSCE201508) and the Jiangsu Key Laboratory of Advanced Design and Manufacturing Technology for Ship at Jiangsu University of Science and Technology (Grant no. CJ1504).

\section{References}

Bellotti, E.C., Franco, L., 2011. Measurement of long waves at the harbor of Marina di Carrara, Italy. Ocean Dynamics 61 (12), 2051-2059. 
Bowers, E.C., 1977. Harbour resonance due to set-down beneath wave groups. Journal of Fluid Mechanics 79, 71-92.

Chen, G.-Y., Chien, C.-C., Su, C.-H., Tseng, H.-M., 2004. Resonance induced by edge waves in Hua-Lien Harbor. Journal of Oceanography 60, 1035-1043.

Chen, Q., 2006. Fully nonlinear Boussinesq-type equations for waves and currents over porous beds. Journal of Engineering Mechanics 132 ( 2), 220-230.

De Jong, M.P.C., Battjes, J.A., 2004. Seiche characteristics of Rotterdam Harbour Coastal Engineering 51 (5-6), 373-386

Dong, G., Gao, J., Ma, X., Wang, G., Ma, Y., 2013. Numerical study of low-frequency waves during harbor resonance. Ocean Engineering 68, 38-46.

Dong, G., Wang, G., Ma, X., Ma, Y., 2010. Harbor resonance induced by subaerial landslide-generated impact waves. Ocean Engineering 37 (10), 927-934.

Dong, J., Wang, B.-L., Liu, H., 2014. Run-up of non-breaking double solitary waves with equal wave heights on a plane beach. Journal of Hydrodynamics 26 (6), 939-950.

El, G.A., Grimshaw, R.H.J., Tiong, W.K., 2012. Transformation of a shoaling undular bore. Journal of Fluid Mechanics 709, 371-395.

Fabrikant, A.L., 1995. Harbor oscillations generated by shear flow. Journal of Fluid Mechanics 282, 203-217.

Gao, J., Ji, C., Gaidai, O., Liu, Y., 2016a. Numerical study of infragravity waves amplification during harbor resonance. Ocean Engineering 116, 90-100.

Gao, J., Ji, C., Gaidai, O., Liu, Y., Ma, X., 2017a. Numerical investigation of transient harbor oscillations induced by N-waves. Coastal Engineering 125, 119-131.

Gao, J., Ji, C., Liu, Y., Gaidai, O., Ma, X., Liu, Z., 2016b. Numerical study on transient harbor oscillations induced by solitary waves. Ocean Engineering 126, 467-480.

Gao, J., Ji, C., Liu, Y., Ma, X., Gaidai, O., 2017b. Influence of offshore topography on the amplification of infragravity oscillations within a harbor. Applied Ocean Research 65, $129-141$.

Gao, J., Ma, X., Dong, G., Wang, G., Ma, Y., 2015. Improvements on the normal mode decomposition method used in harbor resonance. Proceedings of the Institution of Mechanical Engineers, Part M: Journal of Engineering for the Maritime Environment 229 (4), 
$397-410$.

Gao, J., Ma, X., Dong, G., Wang, G., Ma, Y., 2016c. Numerical study of transient harbor resonance induced by solitary waves. Proceedings of the Institution of Mechanical Engineers, Part M: Journal of Engineering for the Maritime Environment 230 (1), 163-176.

Grilli, S.T., Harris, J.C., Shi, F., Kirby, J.T., Bakhsh, T.S.T., Estibals, E., Tehranirad, B., 2012. Numerical modeling of coastal tsunami dissipation and impact, Proceedings of the 33rd International Coastal Engineering Conference (ICCE 2012), Santander (Cantabria), Spain.

Grue, J., Pelinovsky, E.N., Fructus, D., Talipova, T., Kharif, C., 2008. Formation of undular bores and solitary waves in the Strait of Malacca caused by the 26 December 2004 Indian Ocean tsunami. Journal of Geophysical Research 113, C05008, doi:10.1029/2007JC004343.

Kennedy, A.B., Kirby, J.T., Chen, Q., Dalrymple, R.A., 2001. Boussinesq-type equations with improved nonlinear performance. Wave Motion 33, 225-243.

Kumar, P., Zhang, H., Kim, K.I., A.Yuen, D., 2016. Modeling wave and spectral characteristics of moored ship motion in Pohang New Harbor under the resonance conditions. Ocean Engineering 119, 101-113.

López, M., Iglesias, G., 2013. Artificial intelligence for estimating infragravity energy in a harbor. Ocean Engineering 57, 56-63.

López, M., Iglesias, G., 2014. Long wave effects on a vessel at berth. Applied Ocean Research 47, $63-72$.

López, M., Iglesias, G., Kobayashi, N., 2012. Long period oscillations and tidal level in the Port of Ferrol. Applied Ocean Research 38, 126-134.

López, M., López, I., Iglesias, G., 2015. Hindcasting Long Waves in a Port: An ANN Approach. Coastal Engineering Journal 57 (4), 1550019 (20 pages).

Lepelletier, T.G., Raichlen, F., 1987. Harbor oscillations induced by nonlinear transient long waves. Journal of Waterway, Port, Coastal and Ocean Engineering 113 (4), 381-400.

Lo, H.-Y., Park, Y.S., Liu, P.L.-F., 2013. On the run-up and back-wash processes of single and double solitary waves - An experimental study. Coastal Engineering 80, 1-14.

Ma, G., Shi, F., Kirby, J.T., 2012. Shock-capturing non-hydrostatic model for fully dispersive surface wave processes. Ocean Modelling 43-44, 22-35.

Maa, J.P.-Y., Tsai, C.-H., Juang, W.-J., Tseng, H.-M., 2011. A preliminary study on Typhoon Tim 
induced resonance at Hualien Harbor, Taiwan. Ocean Dynamics 61, 411-423.

Madsen, P.A., Fuhrman, D.R., Schäffer, H.A., 2008. On the solitary wave paradigm for tsunamis. Journal of Geophysical Research 113, C12012, doi:10.1029/2008JC004932.

Pattiaratchi, C.B., Wijeratne, E.M.S., 2009. Tide gauge observations of 2004-2007 Indian Ocean tsunamis from Sri Lanka and Western Australia. Pure and Applied Geophysics 166 (1), $233-258$.

Peregrine, D.H., 1966. Calculations of the development of an undular bore. Journal of Fluid Mechanics 25 (2), 321-330.

Pujara, N., Liu, P.L.-F., Yeh, H.H., 2015. An experimental study of the interaction of two successive solitary waves in the swash: A strongly interacting case and a weakly interacting case. Coastal Engineering 105, 66-74.

Rong, Y., Wu, W., Liu, H., 2016. An experimental study of runup of several successive solitary waves of same wave height on slope, Proceedings of the Twenty-sixth (2016) International Ocean and Polar Engineering Conference, Rhodes, Greece, pp. 607-610.

Shi, F., Kirby, J.T., Harris, J.C., Geiman, J.D., Grilli, S.T., 2012. A high-order adaptive time-stepping TVD solver for Boussinesq modeling of breaking waves and coastal inundation. Ocean Modelling 43-44, 36-51.

Sobey, R.J., 2006. Normal mode decomposition for identification of storm tide and tsunami hazard. Coastal Engineering 53, 289-301.

Tadepalli, S., Synolakis, C.E., 1994. The run-up of N-waves on sloping beaches. Proceedings of the Royal Society London A: Mathematical, Physical \& Engineering Sciences 445, 99-112.

Uzaki, K.-i., Matsunaga, N., Nishii, Y., Ikehata, Y., 2010. Cause and countermeasure of long-period oscillations of moored ships and the quantification of surge and heave amplitudes. Ocean Engineering 37, 155-163.

Xuan, R.-T., Wu, W., Liu, H., 2013. An experimental study on runup of two solitary waves on plane beaches. Journal of Hydrodynamics 25 (2), 317-320.

Zhao, X., Wang, B., Liu, H., 2012. Characteristics of tsunami motion and energy budget during runup and rundown processes over a plane beach. Physics of Fluids 24 (6), 062107. 